\title{
Predictive Metabolomic Signatures for Safety Assessment of Metal Oxide Nanoparticles
}

Li Cui ${ }^{\dagger}$, Xiang Wang ${ }^{\ddagger}$, Bingbing Sun ${ }^{\S}$, Tian $\mathrm{Xia}^{*}{ }^{*}$, Shen $\mathrm{Hu}^{*}+\dagger$

${ }^{\dagger}$ School of Dentistry and Jonsson Comprehensive Cancer Center, University of California, Los Angeles, California 90095, United States

Division of NanoMedicine, Department of Medicine, California NanoSystems Institute, University of California, Los Angeles, California 90095, United States

${ }^{\S}$ State Key Laboratory of Fine Chemicals, School of Chemical Engineering, Dalian University of Technology, 2 Linggong Road, 116024, Dalian, China.

Corresponding authors:

Shen Hu, Ph.D., M.B.A., School of Dentistry and Jonsson Comprehensive Cancer Center, University of California, Los Angeles, California 90095, United States, Tel: 310-2068834, Email: shenhu@ucla.edu

Tian Xia, M.D., Ph.D., Division of NanoMedicine, Department of Medicine, California NanoSystems Institute, University of California, Los Angeles, California 90095, United States, Tel: 310-9833359, Email: txia@ucla.edu 
Figure S1
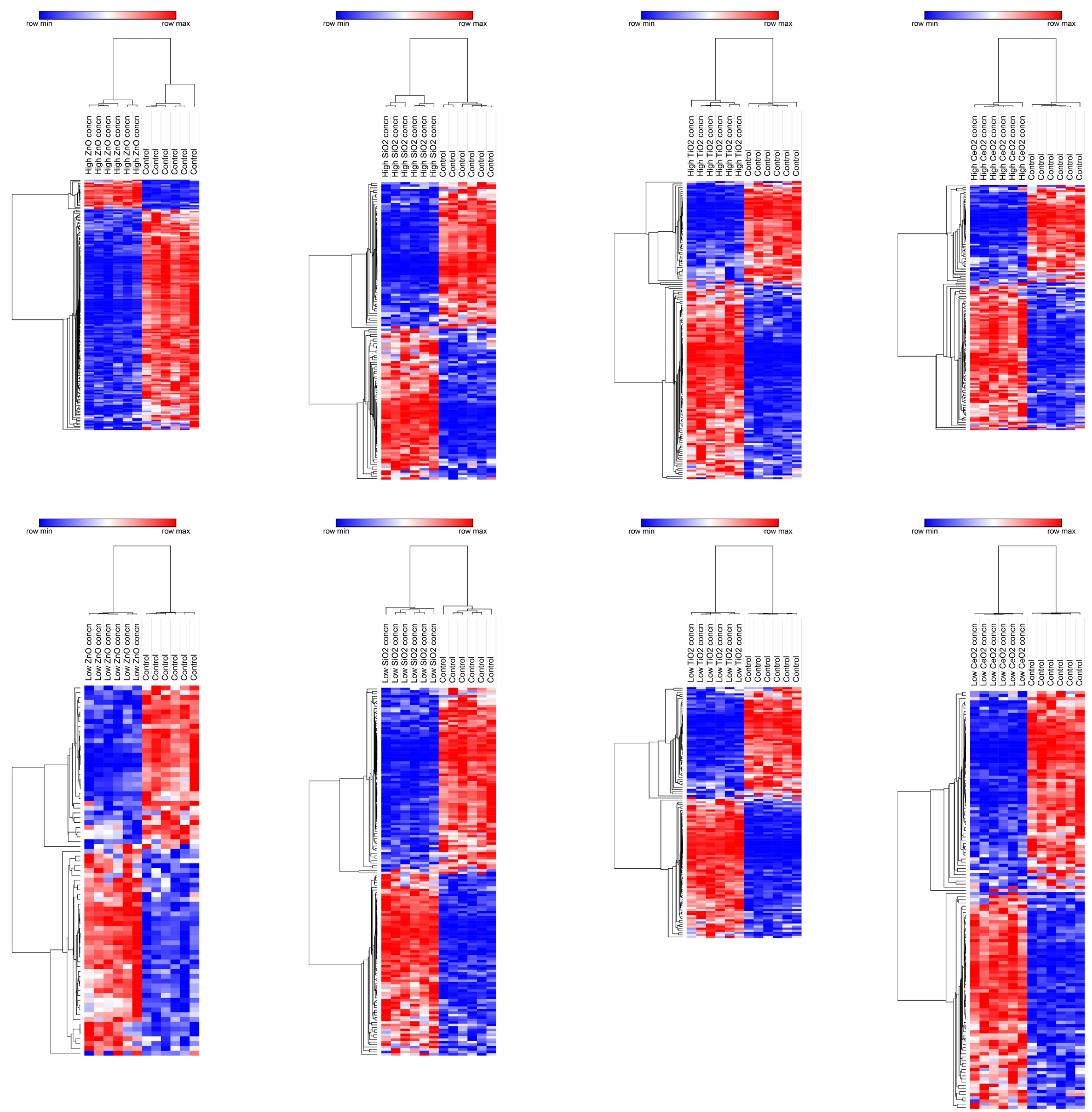

Figure S1. Heatmaps of the identified metabolites with significant alteration between MOx NPs treated and untreated cells at two different concentrations. Each column indicated an independent biological sample and each row represented a metabolite. A range of colors that moved from blue to red indicated lowest to highest level. 


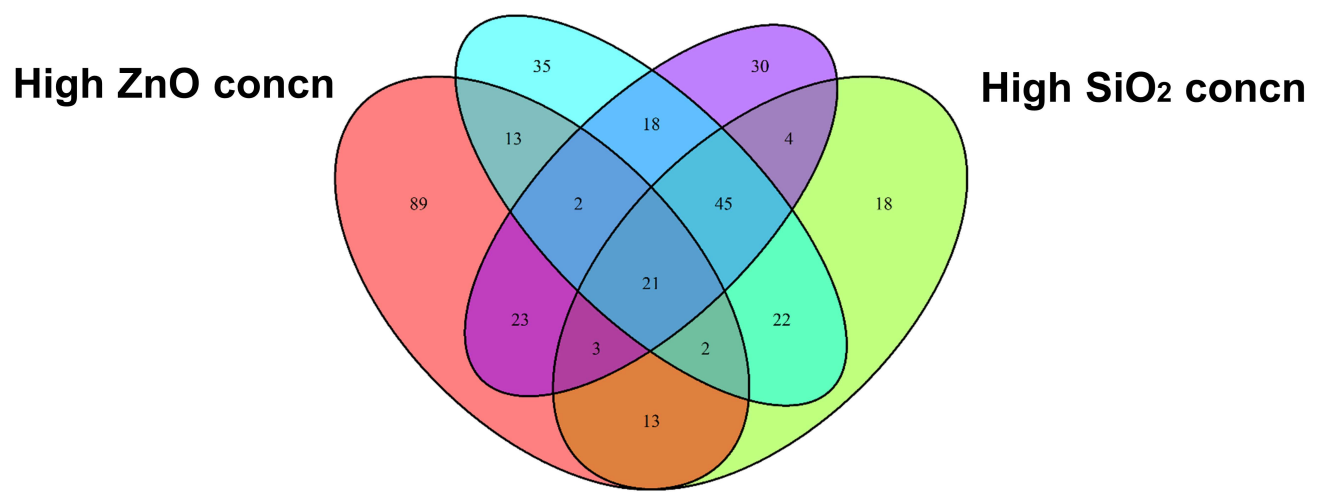

\section{Low $\mathrm{TiO}_{2}$ concn Low $\mathrm{CeO}_{2}$ concn}

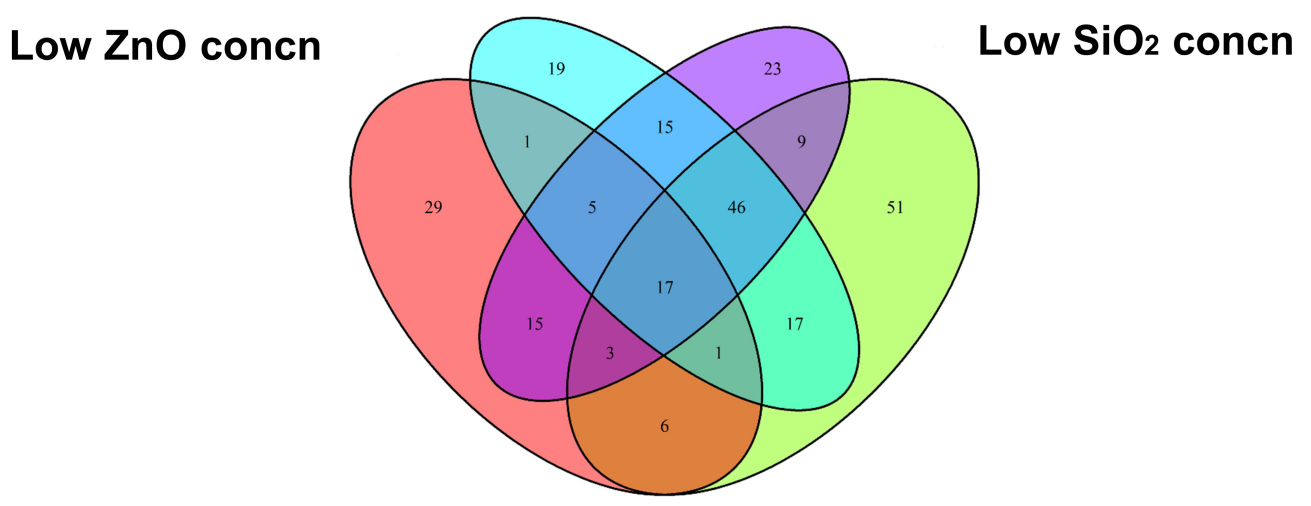

B

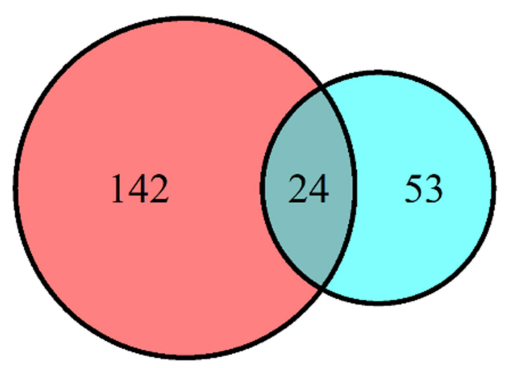

High ZnO concn

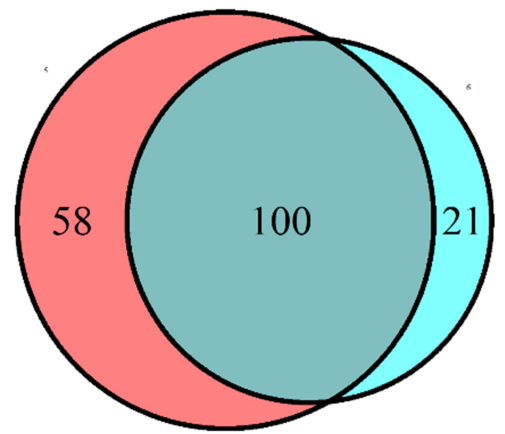

High $\mathrm{TiO}_{2}$ conen Low $\mathrm{TiO}_{2}$ conen

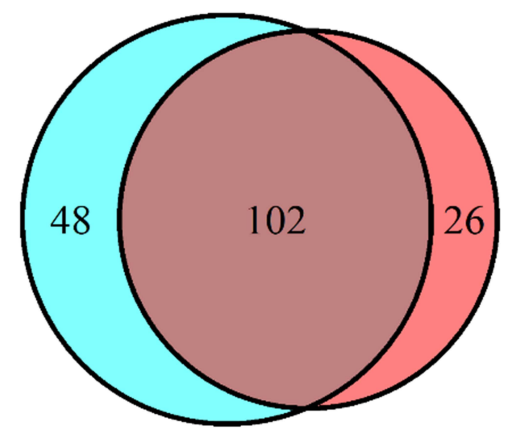

Low $\mathrm{SiO}_{2}$ concn High $\mathrm{SiO}_{2}$ conen

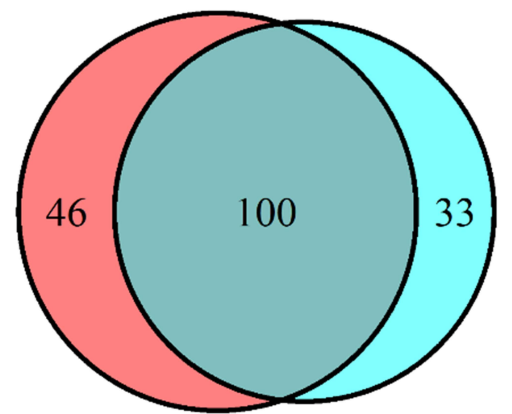

High $\mathrm{CeO}_{2}$ concn Low $\mathrm{CeO}_{2}$ concn

Figure S2. The commonly changed and differentially altered metabolites among different groups. (A) Totally 22 or 17 commonly altered metabolites were detected among four types of MOx NPs at the high or low concentrations, respectively. Each type of MOx NPs had its own specific metabolomic alterations. (B) A total of 102, 100 and 100 commonly altered metabolites were found between high and low concentration $\mathrm{SiO}_{2} \mathrm{NP}$ groups, between high and low concentration $\mathrm{TiO}_{2} \mathrm{NP}$ groups, and between high and low concentration $\mathrm{CeO}_{2} \mathrm{NP}$ groups, respectively. Only 24 metabolites were commonly altered between high and low concentration $\mathrm{ZnO} \mathrm{NP}$ groups. 
Figure S3

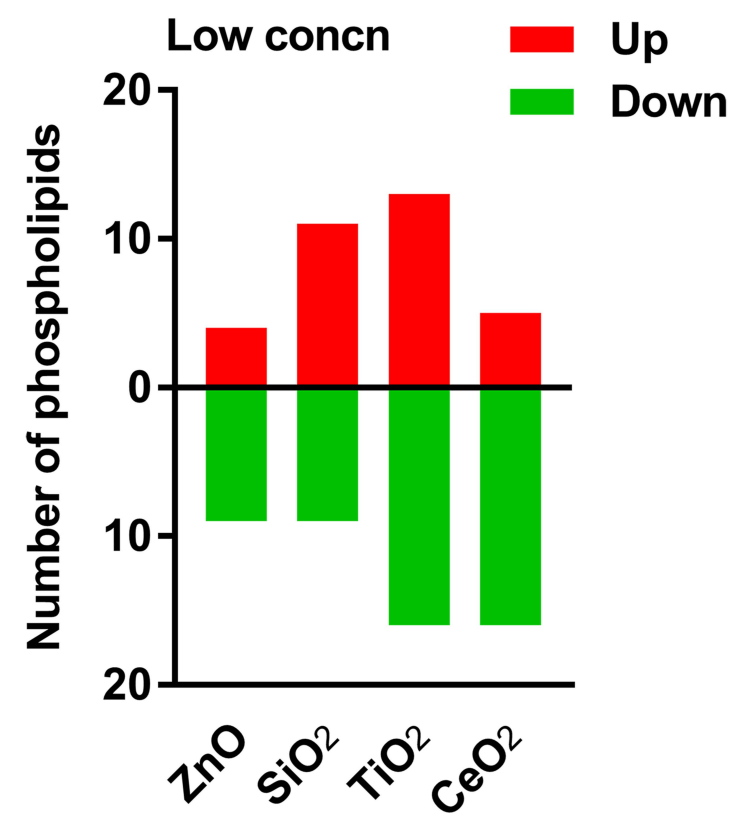

Figure S3. The number of significantly altered phospholipids in bronchial epithelial cells subjected to four types of MOx NPs treatment at low concentration. 
Figure S6

A

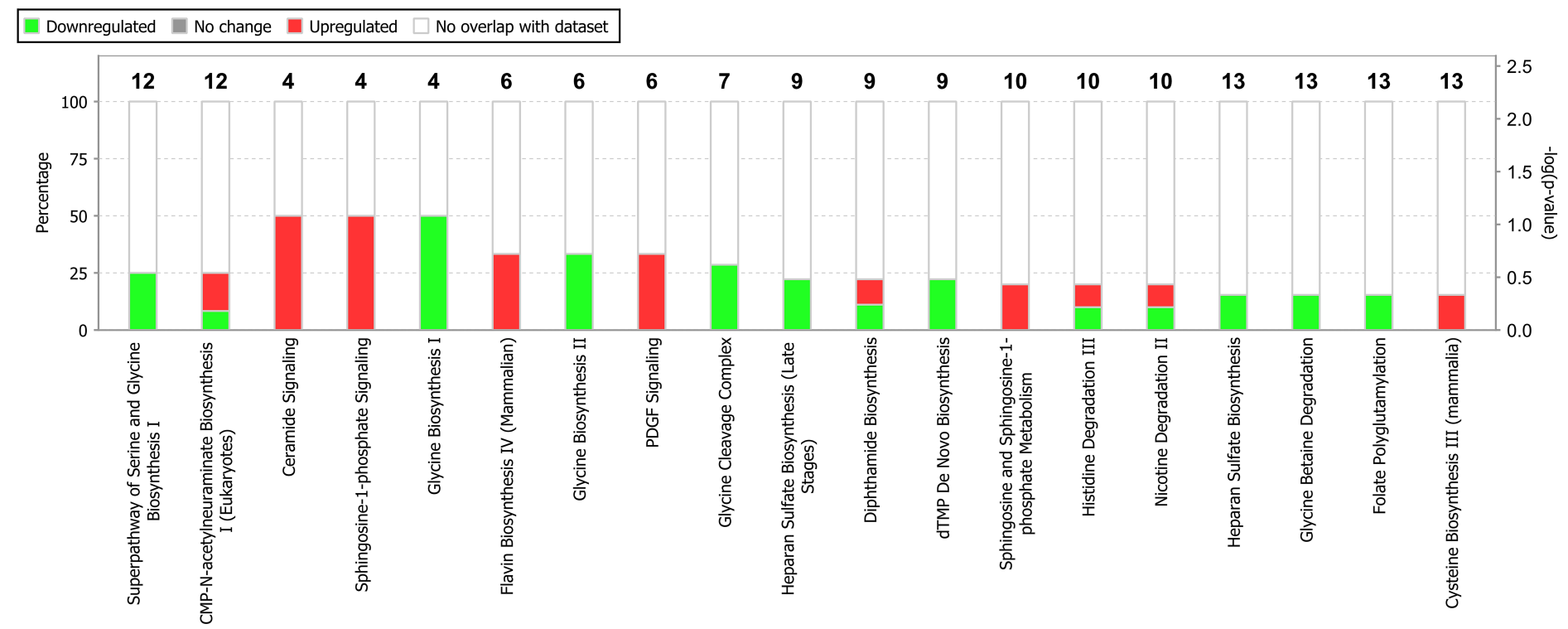

High $\mathrm{TiO}_{2}$ concn

B

$\square$ Downregulated $\quad$ No change $\quad$ Upregulated $\square$ No overlap with dataset

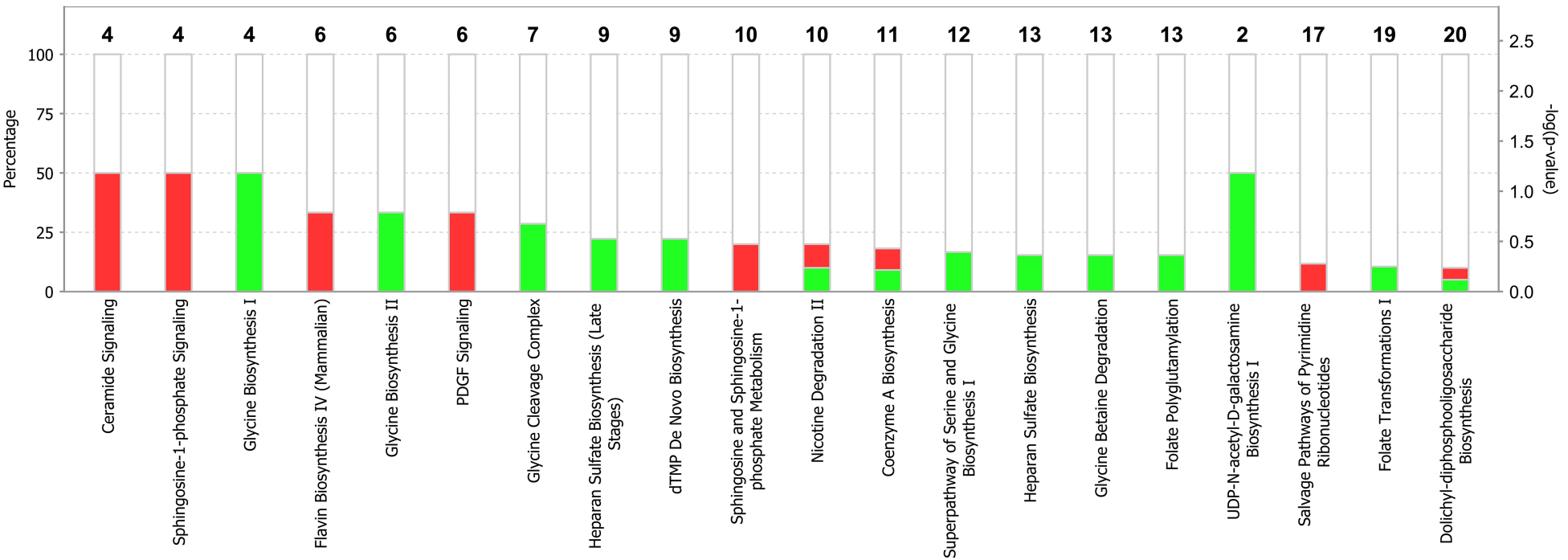

Low $\mathrm{TiO}_{2}$ concn

Figure S6. IPA analysis revealed the significantly affected metabolic pathways in the bronchial epithelial cells subjected to high (A) or low (B) concentration $\mathrm{TiO}_{2} \mathrm{NPs}$ treatment. 
Figure S7

\section{A}

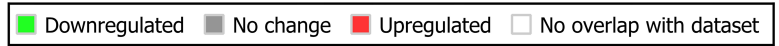

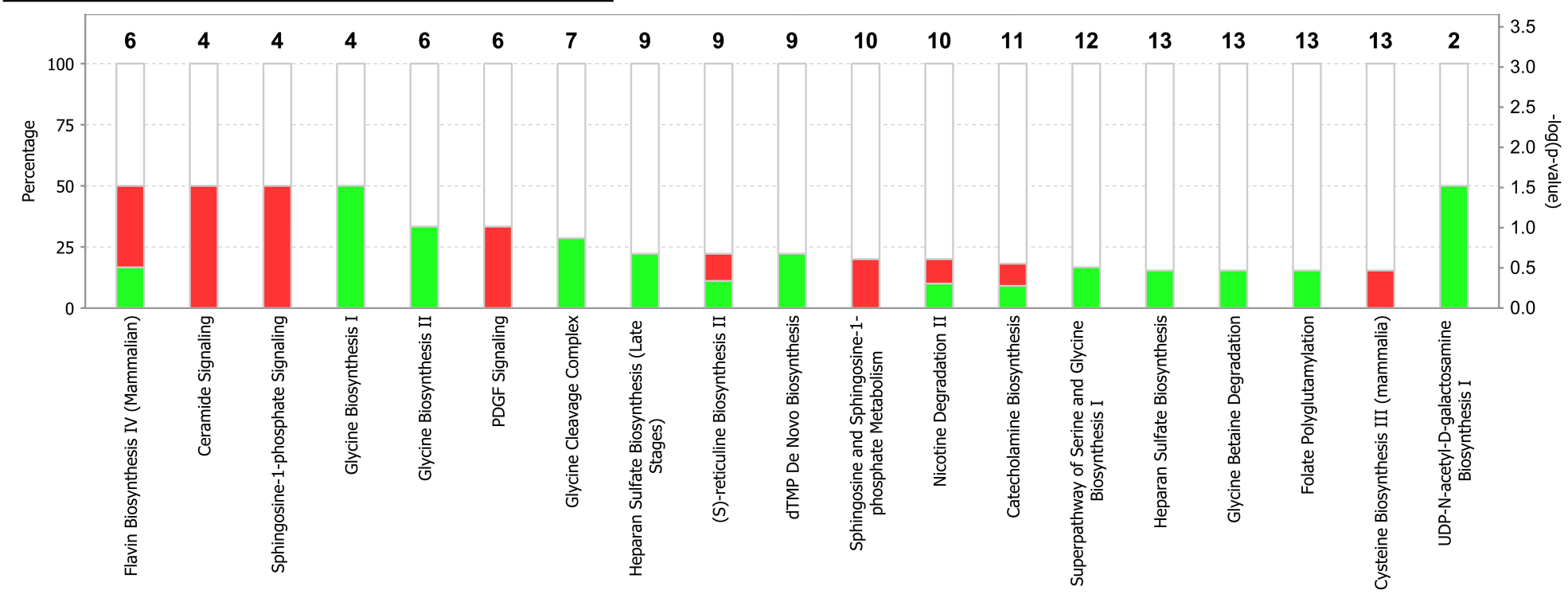

B

High $\mathrm{CeO}_{2}$ concn

Downregulated $\quad$ No change $\quad$ U Upregulated $\square$ No overlap with dataset

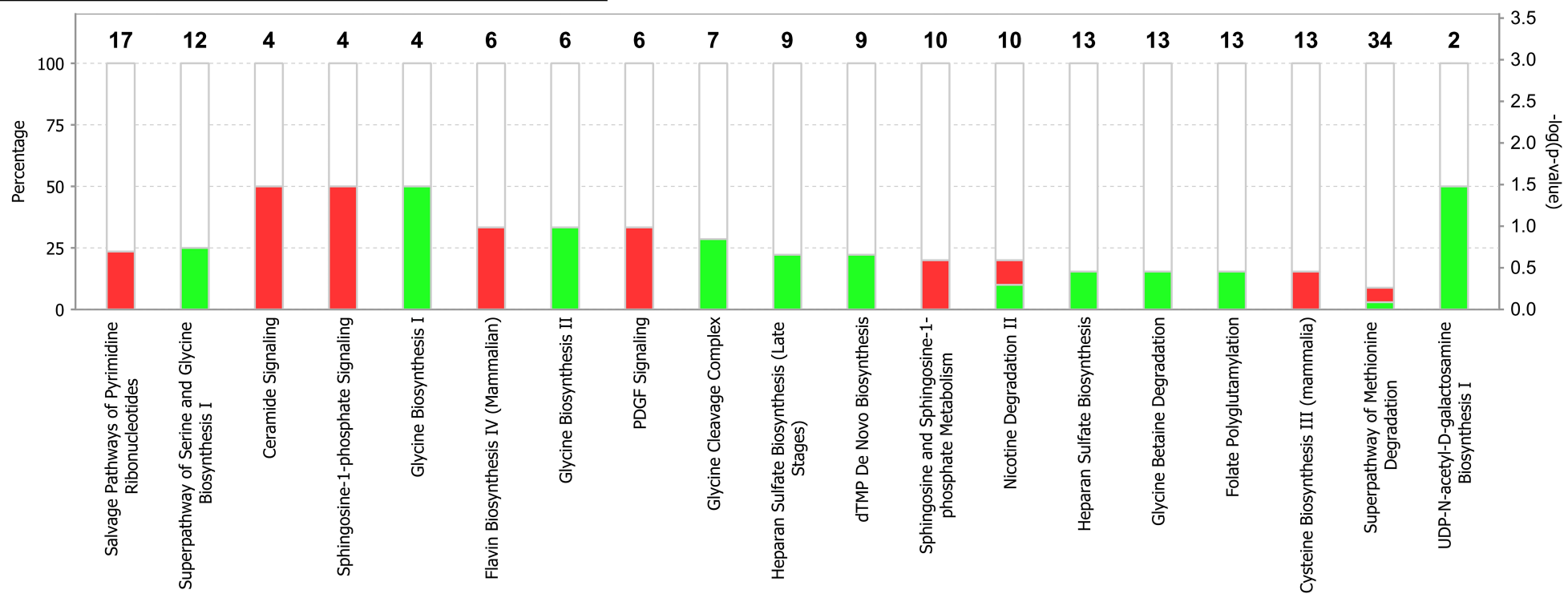

Low $\mathrm{CeO}_{2}$ concn

Figure S7. IPA analysis revealed the significantly affected metabolic pathways in the bronchial epithelial cells subjected to high (A) or low (B) concentration $\mathrm{CeO}_{2}$ NPs treatment. 
A

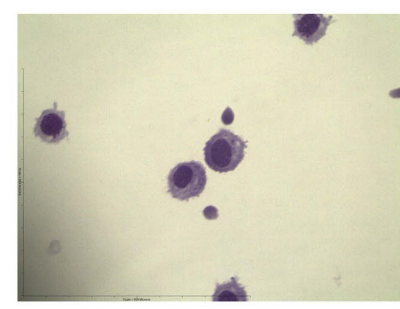

CTRL

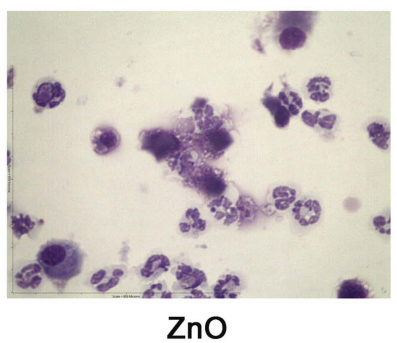

$\mathrm{ZnO}$

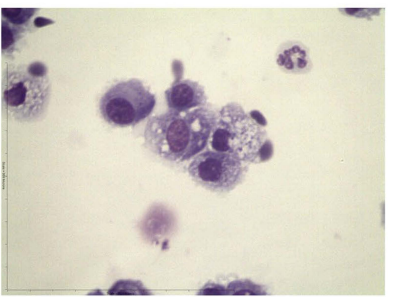

$\mathrm{SiO}_{2}$

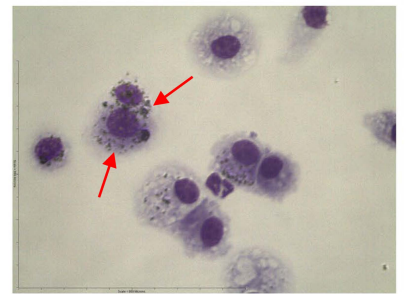

$\mathrm{TiO}_{2}$

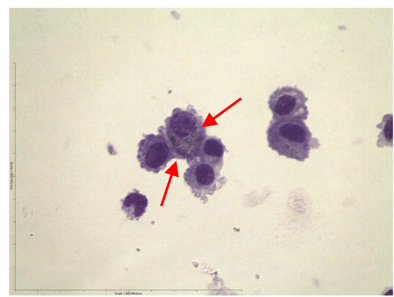

$\mathrm{CeO}_{2}$

B
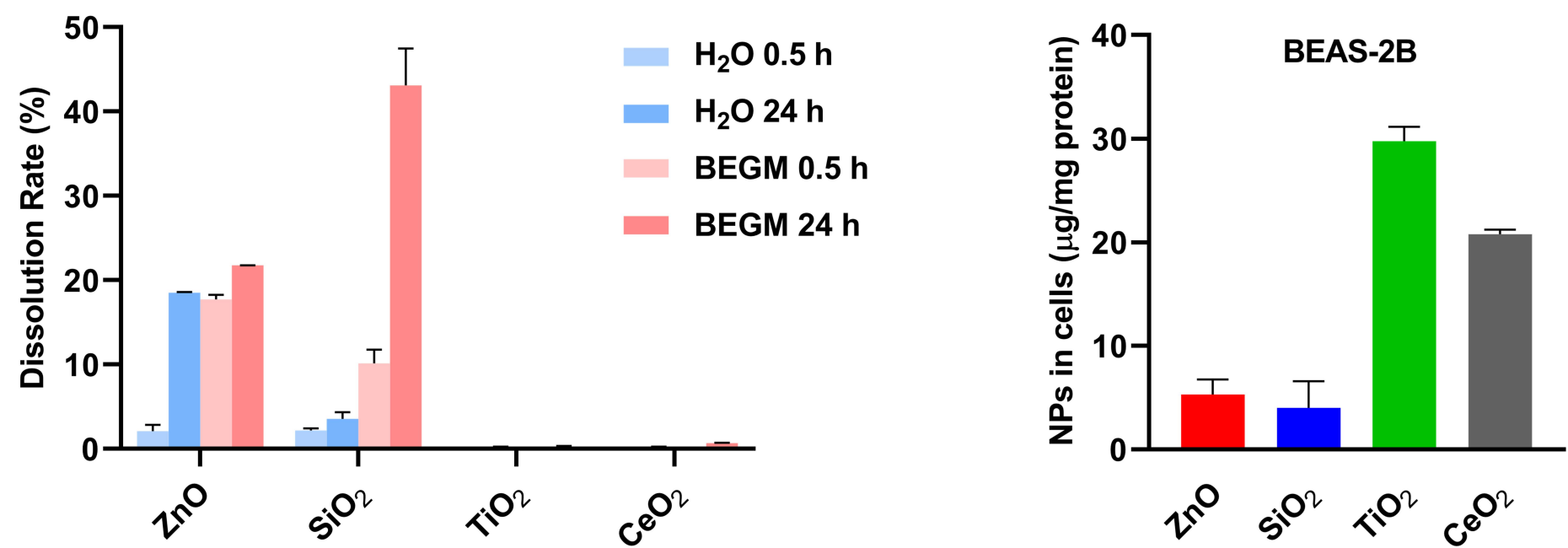

Figure S8. Cellular uptake and the dissolution of MOx nanoparticles. (A) Significant uptake of MOx NPs was observed in mouse alveolar macrophages. (B) Nanoparticle dissolution in cell culture medium. The particle suspensions were prepared in DI water or cell culture medium at concentration of $100 \mu \mathrm{g} / \mathrm{mL}$. The particle suspensions were incubated at $37^{\circ} \mathrm{C}$. Samples were collected at 0.5 and $24 \mathrm{~h}$, centrifuged at $15000 \mathrm{rpm}$, and each supernatant was collected for acid digestion and ICP-OES analysis. (C) Use of ICP-OES to quantify MOx nanoparticle uptake in BEAS-2B cells. After $4 \mathrm{~h}$ of treatment with $12.5 \mu \mathrm{g} / \mathrm{mL}$ of the selected nanoparticles, BEAS2B cell pellets were collected and acid digested for assessment of their metal content by ICP-OES. 


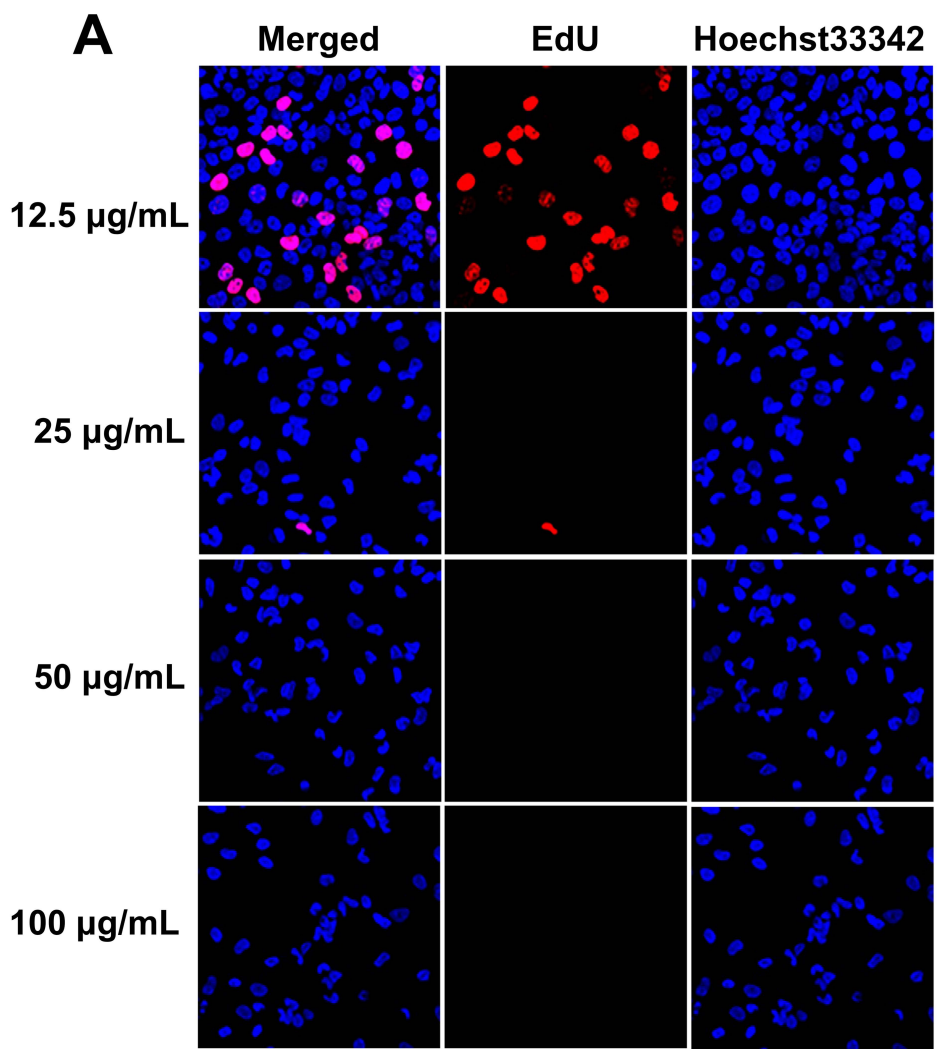

C

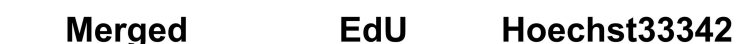

$12.5 \mu \mathrm{g} / \mathrm{mL}$

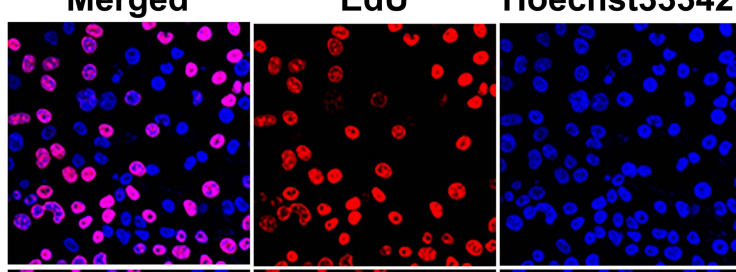

$25 \mu \mathrm{g} / \mathrm{mL}$

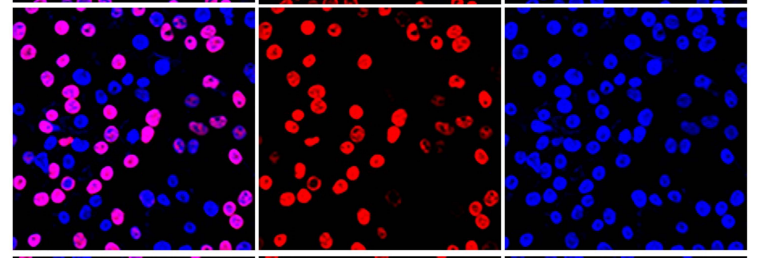

$50 \mu \mathrm{g} / \mathrm{mL}$

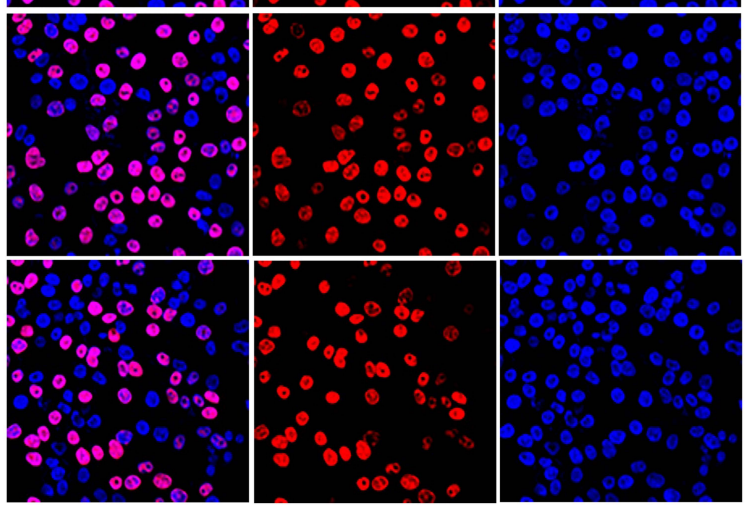

$\mathrm{TiO}_{2}$

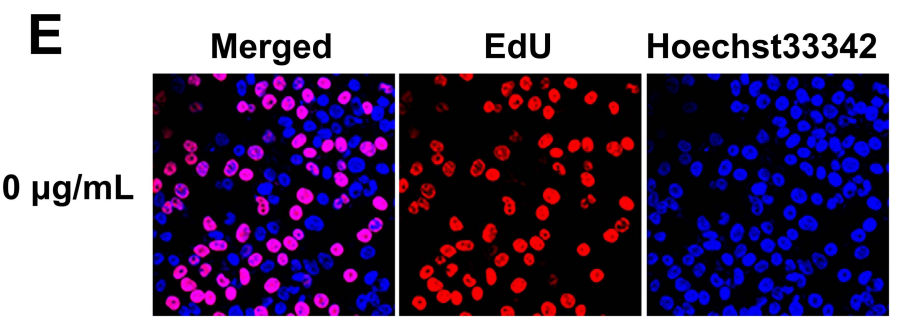

CTRL

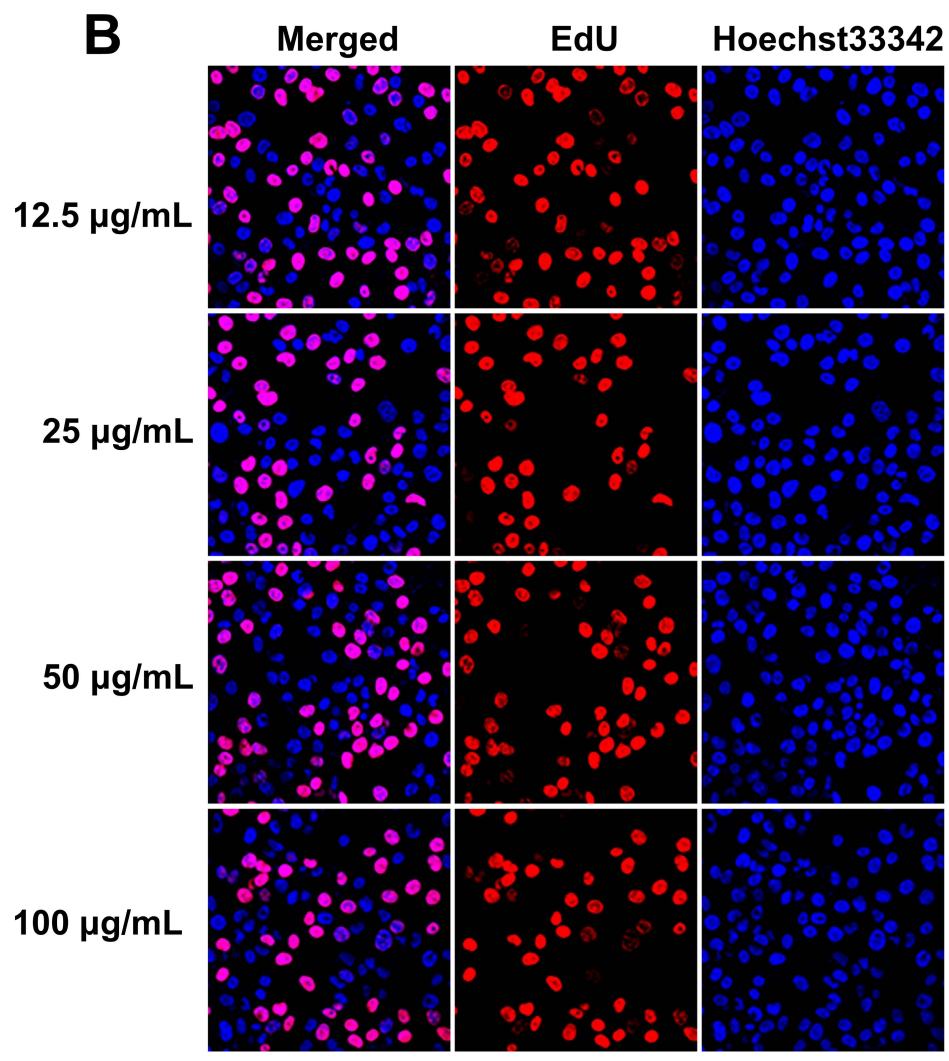

$\mathrm{SiO}_{2}$

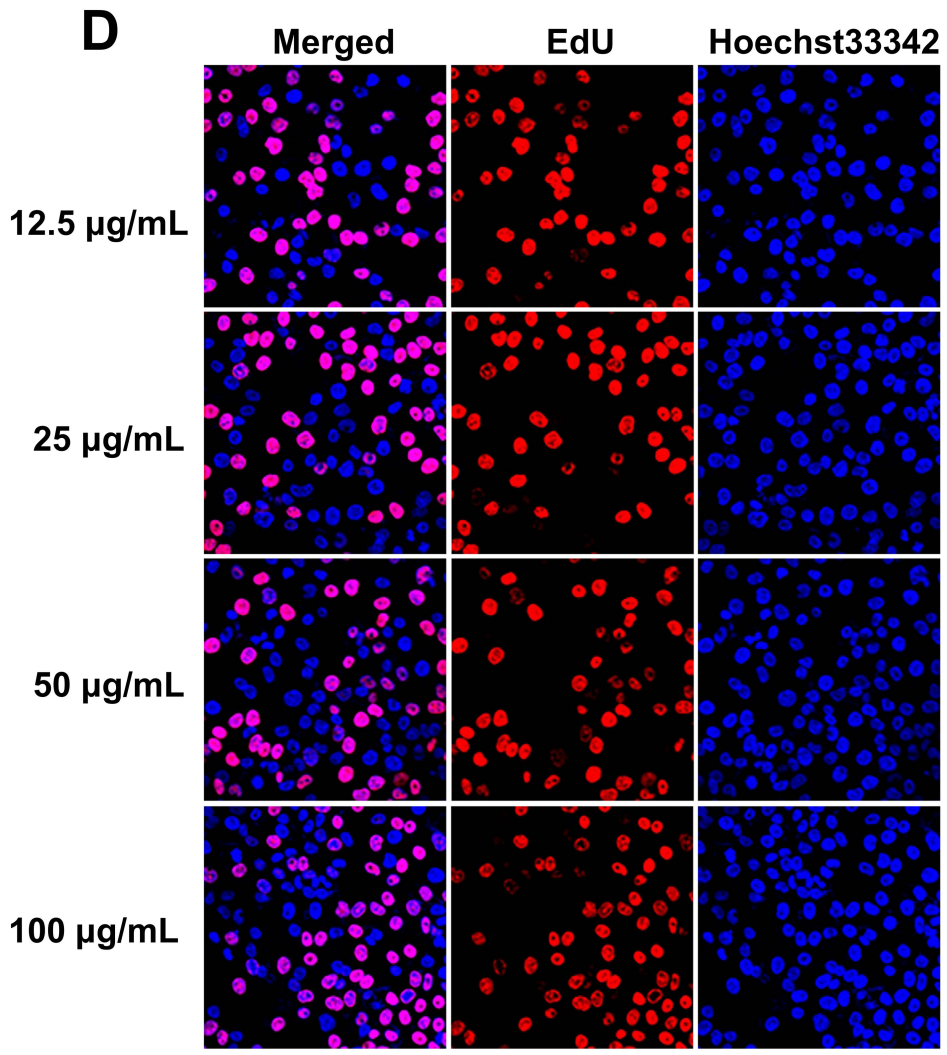

$\mathrm{CeO}_{2}$

Figure S9. The effects of MOx NPs on the proliferation of bronchial epithelial cells. (A) The percentage of EdU positive cells was significantly lower in ZnO NPs treated group at the concentration of $12.5 \mu \mathrm{g} / \mathrm{mL}, 25 \mu \mathrm{g} / \mathrm{mL}, 50$ $\mu \mathrm{g} / \mathrm{mL}$ and $100 \mu \mathrm{g} / \mathrm{mL}$ compared to that in the control group $(0 \mu \mathrm{g} / \mathrm{mL})$. No significant change was found for the percentage of EdU positive cells at the above four concentration between $\mathrm{SiO}_{2} \mathrm{NPs}$ treated group and the control group (B), between $\mathrm{TiO}_{2} \mathrm{NPs}$ treated group and the control group (C), and between $\mathrm{CeO}_{2} \mathrm{NPs}$ treated group and the control group (D). (E) The percentage of EdU positive cells in the control group. 
Figure S10

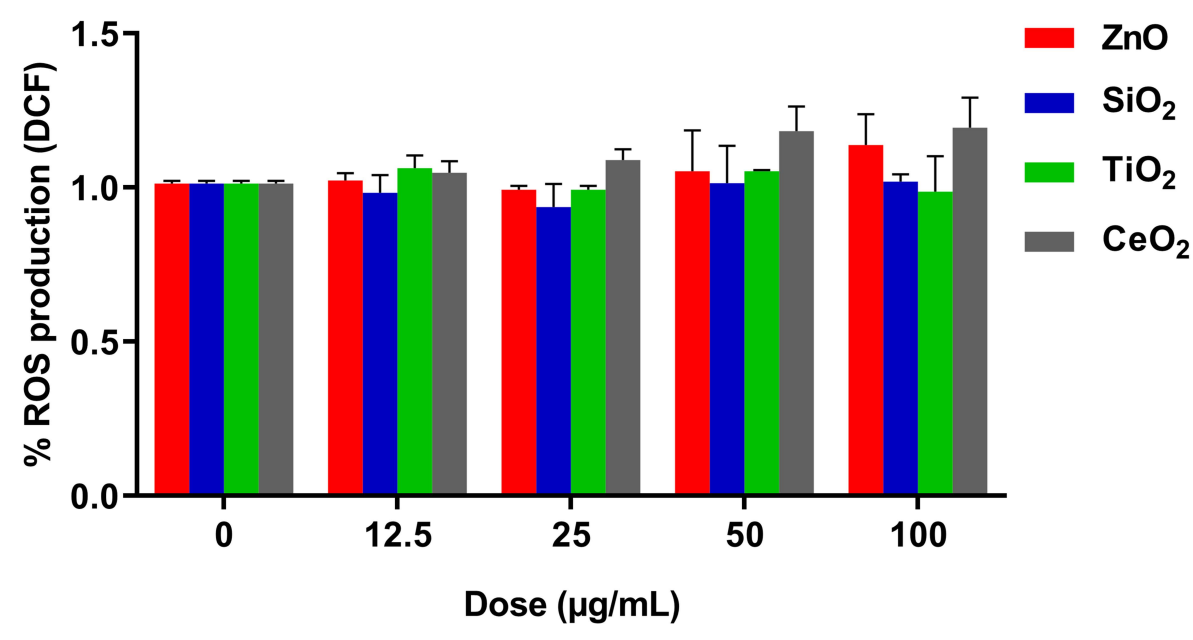

Figure S10. No statistical significant difference was found for the ROS production in bronchial epithelial cells subjected to four types of NPs exposure at the concentration ranging from $0 \mu \mathrm{g} / \mathrm{mL}-100 \mu \mathrm{g} / \mathrm{mL}$. 


\begin{tabular}{|c|c|c|c|}
\hline $\begin{array}{l}\text { Supplementa } \\
\text { treated with } 1\end{array}$ & $\begin{array}{l}\text { Table 1. Significantly altered } \\
\text {-dose ZnO NPs. }\end{array}$ & & S-2B cells \\
\hline ID & Description & Ratio & q value \\
\hline HMDB00034 & Adenine & 0.529 & $3.582 \mathrm{E}-04$ \\
\hline HMDB00045 & Adenosine monophosphate & 0.256 & $1.034 \mathrm{E}-09$ \\
\hline HMDB00050 & Adenosine & 0.505 & $2.587 \mathrm{E}-05$ \\
\hline HMDB00052 & Argininosuccinic acid & 0.399 & $6.039 \mathrm{E}-09$ \\
\hline HMDB00058 & Cyclic AMP & 0.252 & $3.352 \mathrm{E}-07$ \\
\hline HMDB00062 & L-Carnitine & 0.311 & $5.497 \mathrm{E}-10$ \\
\hline HMDB00064 & Creatine & 0.413 & $2.171 \mathrm{E}-07$ \\
\hline HMDB00072 & cis-Aconitic acid & 0.123 & $1.086 \mathrm{E}-03$ \\
\hline HMDB00086 & Glycerophosphocholine & 0.651 & $7.739 \mathrm{E}-08$ \\
\hline HMDB00094 & Citric acid & 0.209 & $2.923 \mathrm{E}-07$ \\
\hline HMDB00125 & Glutathione & 0.059 & $3.383 \mathrm{E}-04$ \\
\hline HMDB00148 & L-Glutamic acid & 0.348 & $1.160 \mathrm{E}-08$ \\
\hline HMDB00158 & L-Tyrosine & 0.480 & $8.648 \mathrm{E}-04$ \\
\hline HMDB00159 & L-Phenylalanine & 0.511 & $4.691 \mathrm{E}-06$ \\
\hline HMDB00161 & L-Alanine & 0.394 & $1.817 \mathrm{E}-08$ \\
\hline HMDB00167 & L-Threonine & 0.450 & $5.832 \mathrm{E}-07$ \\
\hline HMDB00177 & L-Histidine & 0.416 & $2.480 \mathrm{E}-05$ \\
\hline HMDB00191 & L-Aspartic acid & 0.757 & $6.412 \mathrm{E}-06$ \\
\hline HMDB00205 & Phenylpyruvic acid & 0.616 & $8.673 \mathrm{E}-06$ \\
\hline HMDB00210 & Pantothenic acid & 0.274 & $7.449 \mathrm{E}-05$ \\
\hline HMDB00226 & Orotic acid & 0.329 & $7.536 \mathrm{E}-04$ \\
\hline HMDB00230 & N-Acetylneuraminic acid & 3.439 & $7.048 \mathrm{E}-08$ \\
\hline HMDB00251 & Taurine & 0.377 & $1.844 \mathrm{E}-11$ \\
\hline HMDB00265 & Liothyronine & 0.669 & $5.320 \mathrm{E}-07$ \\
\hline HMDB00280 & Phosphoribosyl pyrophosphate & 3.641 & $9.337 \mathrm{E}-06$ \\
\hline HMDB00288 & Uridine 5'-monophosphate & 0.526 & $2.256 \mathrm{E}-06$ \\
\hline HMDB00295 & Uridine 5'-diphosphate & 0.662 & $1.020 \mathrm{E}-02$ \\
\hline HMDB00403 & 2-Hydroxyadenine & 1.737 & $6.530 \mathrm{E}-06$ \\
\hline HMDB00536 & Adenylsuccinic acid & 0.209 & $2.456 \mathrm{E}-03$ \\
\hline HMDB00562 & Creatinine & 0.359 & $3.645 \mathrm{E}-09$ \\
\hline HMDB00651 & Decanoylcarnitine & 0.362 & $1.212 \mathrm{E}-04$ \\
\hline HMDB00656 & Cysteineglutathione disulfide & 0.369 & $9.670 \mathrm{E}-06$ \\
\hline HMDB00687 & L-Leucine & 0.457 & $1.323 \mathrm{E}-07$ \\
\hline HMDB00695 & Ketoleucine & 3.525 & $1.370 \mathrm{E}-07$ \\
\hline HMDB00734 & Indoleacrylic acid & 0.447 & $1.212 \mathrm{E}-07$ \\
\hline HMDB00735 & Hydroxyphenylacetylglycine & 0.511 & $7.130 \mathrm{E}-05$ \\
\hline HMDB00744 & Malic acid & 0.053 & $1.935 \mathrm{E}-05$ \\
\hline HMDB00812 & N-Acetyl-L-aspartic acid & 0.319 & $1.062 \mathrm{E}-03$ \\
\hline HMDB00825 & 3'-Sialyllactose/6'-Sialyllactose & 0.067 & $1.619 \mathrm{E}-06$ \\
\hline HMDB00828 & Ureidosuccinic acid & 0.159 & $2.190 \mathrm{E}-06$ \\
\hline HMDB00905 & Deoxyadenosine monophosphate & 0.267 & $2.181 \mathrm{E}-02$ \\
\hline
\end{tabular}




\begin{tabular}{|c|c|c|c|}
\hline HMDB00929 & L-Tryptophan & 0.489 & $4.421 \mathrm{E}-06$ \\
\hline HMDB00935 & Uridine diphosphate glucuronic acid & 0.390 & $3.540 \mathrm{E}-06$ \\
\hline HMDB00939 & S-Adenosylhomocysteine & 0.313 & $3.155 \mathrm{E}-04$ \\
\hline HMDB00943 & Threonic acid & 4.058 & $1.283 \mathrm{E}-03$ \\
\hline HMDB00988 & S-Adenosylmethioninamine & 0.118 & $1.766 \mathrm{E}-03$ \\
\hline HMDB01014 & 4-Imidazolone-5-propionic acid & 2.141 & $4.135 \mathrm{E}-07$ \\
\hline HMDB01066 & S-Lactoylglutathione & 0.186 & $1.910 \mathrm{E}-03$ \\
\hline HMDB01068 & D-Sedoheptulose 7-phosphate & 3.066 & $1.841 \mathrm{E}-05$ \\
\hline HMDB01095 & GDP-L-fucose & 0.503 & $1.383 \mathrm{E}-05$ \\
\hline HMDB01117 & 4-Phosphopantothenoylcysteine & 0.102 & $1.155 \mathrm{E}-04$ \\
\hline HMDB01138 & N-Acetylglutamic acid & 0.101 & $2.676 \mathrm{E}-07$ \\
\hline HMDB01142 & FMNH2 & 0.342 & $4.431 \mathrm{E}-04$ \\
\hline HMDB01168 & 2,4-Decadienoyl-CoA & 0.245 & $7.121 \mathrm{E}-05$ \\
\hline HMDB01178 & Adenosine diphosphate ribose & 0.177 & $1.427 \mathrm{E}-03$ \\
\hline HMDB01202 & dCMP & 0.376 & $1.070 \mathrm{E}-04$ \\
\hline HMDB01227 & 5-Thymidylic acid & 0.087 & $1.663 \mathrm{E}-05$ \\
\hline HMDB01263 & Allysine & 0.325 & 4.169E-02 \\
\hline HMDB01341 & ADP & 0.442 & $5.935 \mathrm{E}-05$ \\
\hline HMDB01397 & Guanosine monophosphate & 0.114 & $1.018 \mathrm{E}-04$ \\
\hline HMDB01406 & Niacinamide & 0.149 & $1.844 \mathrm{E}-11$ \\
\hline HMDB01409 & dUMP & 0.064 & $6.544 \mathrm{E}-05$ \\
\hline HMDB01489 & Ribose-1-phosphate & 0.580 & 4.938E-08 \\
\hline HMDB01546 & $\mathrm{CDP}$ & 0.641 & $3.532 \mathrm{E}-06$ \\
\hline HMDB01586 & Glucose-6-phosphate & 0.083 & $3.566 \mathrm{E}-06$ \\
\hline HMDB01867 & 4-Aminohippuric acid & 0.550 & $3.535 \mathrm{E}-08$ \\
\hline HMDB02007 & Tetracosahexaenoic acid & 0.601 & $6.890 \mathrm{E}-05$ \\
\hline HMDB02013 & Butyrylcarnitine & 0.196 & $8.430 \mathrm{E}-10$ \\
\hline HMDB02014 & cis-5-Tetradecenoylcarnitine & 0.393 & $2.183 \mathrm{E}-09$ \\
\hline HMDB02062 & Norepinephrine sulfate & 0.636 & $7.179 \mathrm{E}-07$ \\
\hline HMDB02064 & N-Acetylputrescine & 0.686 & $1.740 \mathrm{E}-06$ \\
\hline HMDB02135 & S-(3-oxo-3-carboxy-n-propyl)cysteine & 2.104 & $3.529 \mathrm{E}-03$ \\
\hline HMDB02250 & Dodecanoylcarnitine & 0.219 & $2.775 \mathrm{E}-05$ \\
\hline HMDB02639 & Sulfolithocholylglycine & 0.456 & $8.212 \mathrm{E}-04$ \\
\hline HMDB02664 & Prostaglandin E2 & 0.532 & $7.189 \mathrm{E}-03$ \\
\hline HMDB02666 & Thiamine monophosphate & 0.198 & $7.521 \mathrm{E}-04$ \\
\hline HMDB02817 & N-Acetylglucosamine 6-phosphate & 1.743 & $1.244 \mathrm{E}-06$ \\
\hline HMDB02984 & 11-Hydroxyandrosterone & 0.009 & $4.820 \mathrm{E}-07$ \\
\hline HMDB03152 & N-Methylnicotinamide & 5.930 & $3.359 \mathrm{E}-04$ \\
\hline HMDB03229 & Palmitoleic acid & 0.579 & $1.734 \mathrm{E}-04$ \\
\hline HMDB03269 & Nicotinuric acid & 0.711 & $6.955 \mathrm{E}-0$ \\
\hline HMDB03349 & L-Dihydroorotic acid & 0.624 & $4.375 \mathrm{E}-0$ \\
\hline HMDB03447 & Tryptophanol & 0.494 & $2.131 \mathrm{E}-0$ \\
\hline HMDB03464 & 4-Guanidinobutanoic acid & 2.193 & $5.725 \mathrm{E}-\mathrm{C}$ \\
\hline HMDB03892 & (S)-N-Methylsalsolinol & 4.102 & $4.047 \mathrm{E}-\mathrm{C}$ \\
\hline
\end{tabular}




\begin{tabular}{|c|c|c|c|}
\hline HMDB04186 & 3-Methyldioxyindole & 0.513 & $1.490 \mathrm{E}-06$ \\
\hline HMDB04220 & Biotinyl-5'-AMP & 0.491 & $1.895 \mathrm{E}-04$ \\
\hline HMDB04326 & $\begin{array}{l}\text { 2'-O-Methyladenosine/ } \\
\text { 3'-O-Methyladenosine }\end{array}$ & 1.629 & 3.004E-04 \\
\hline HMDB04666 & 2-Arachidonylglycerol & 0.656 & $3.020 \mathrm{E}-05$ \\
\hline HMDB04976 & Glucosylceramide (d18:1/26:1(17Z)) & 0.070 & $2.662 \mathrm{E}-07$ \\
\hline HMDB04977 & Glucosylceramide (d18:1/26:0) & 0.505 & $8.086 \mathrm{E}-07$ \\
\hline HMDB05066 & Tetradecanoylcarnitine & 0.326 & 4.667E-04 \\
\hline HMDB05085 & Leukotriene B4 dimethylamide & 0.210 & $1.447 \mathrm{E}-02$ \\
\hline HMDB06029 & N-Acetylglutamine & 0.203 & $3.408 \mathrm{E}-04$ \\
\hline HMDB06070 & Pregnanetriol & 0.678 & 5.884E-05 \\
\hline HMDB06210 & Heptadecanoyl carnitine & 0.651 & $7.262 \mathrm{E}-07$ \\
\hline HMDB06319 & $\begin{array}{l}\text { Alpha-linolenyl carnitine/ } \\
\text { Gamma-linolenyl carnitine }\end{array}$ & 0.443 & $4.553 \mathrm{E}-05$ \\
\hline HMDB06462 & Homocysteinesulfinic acid & 0.141 & $1.404 \mathrm{E}-03$ \\
\hline HMDB06730 & QYNAD & 0.445 & $2.554 \mathrm{E}-03$ \\
\hline HMDB06831 & 3-Dehydroxycarnitine & 0.673 & 4.399E-06 \\
\hline HMDB06944 & 1,4-beta-D-Glucan & 0.204 & $9.365 \mathrm{E}-03$ \\
\hline HMDB07877 & $\operatorname{PC}(14: 0 / 18: 4)$ & 0.281 & $5.201 \mathrm{E}-06$ \\
\hline HMDB08156 & $\operatorname{PC}(18: 2 / 22: 6)$ & 0.274 & $2.005 \mathrm{E}-03$ \\
\hline HMDB08786 & $\mathrm{PC}(24: 0 / \mathrm{P}-18: 1)$ & 0.326 & 5.274E-08 \\
\hline HMDB09144 & $\operatorname{PE}(18: 3 / 22: 6)$ & 0.145 & $6.234 \mathrm{E}-03$ \\
\hline HMDB09542 & $\operatorname{PE}(22: 1 / 24: 1)$ & 0.090 & 7.923E-06 \\
\hline HMDB09819 & $\mathrm{PI}(18: 0 / 22: 5)$ & 0.227 & 4.141E-02 \\
\hline HMDB10364 & 11-Hydroxyprogesterone 11-glucuronide & 0.270 & $1.654 \mathrm{E}-02$ \\
\hline HMDB10379 & LysoPC(14:0) & 0.626 & 5.844E-07 \\
\hline HMDB10380 & LysoPC(14:1) & 0.579 & $5.485 \mathrm{E}-07$ \\
\hline HMDB10383 & LysoPC(16:1) & 0.574 & 4.629E-07 \\
\hline HMDB10387 & LysoPC(18:3) & 0.315 & $2.182 \mathrm{E}-08$ \\
\hline HMDB10715 & 2-Phenylacetamide & 0.491 & $1.352 \mathrm{E}-08$ \\
\hline HMDB11171 & L-gamma-Glutamyl-L-leucine & 0.549 & $1.174 \mathrm{E}-06$ \\
\hline HMDB11470 & LysoPE(14:0) & 0.629 & $4.557 \mathrm{E}-04$ \\
\hline HMDB11474 & LysoPE(16:1) & 0.488 & $1.288 \mathrm{E}-03$ \\
\hline HMDB11477 & LysoPE(18:2) & 0.609 & $3.731 \mathrm{E}-06$ \\
\hline HMDB11478 & LysoPE(18:3) & 0.645 & $1.620 \mathrm{E}-06$ \\
\hline HMDB11484 & LysoPE(20:3) & 0.655 & $1.137 \mathrm{E}-03$ \\
\hline HMDB11487 & LysoPE(20:4) & 0.580 & $1.714 \mathrm{E}-06$ \\
\hline HMDB11489 & LysoPE(20:5) & 0.601 & $1.216 \mathrm{E}-05$ \\
\hline HMDB11494 & LysoPE(22:5) & 0.607 & $2.128 \mathrm{E}-05$ \\
\hline HMDB11499 & LysoPE(24:6) & 0.296 & $3.874 \mathrm{E}-05$ \\
\hline HMDB11526 & LysoPE(22:6) & 0.643 & $2.573 \mathrm{E}-04$ \\
\hline HMDB11533 & $\operatorname{MG}(16: 0)$ & 0.440 & $3.450 \mathrm{E}-07$ \\
\hline HMDB11534 & $\operatorname{MG}(16: 1)$ & 0.577 & $2.366 \mathrm{E}-07$ \\
\hline HMDB11536 & $\operatorname{MG}(18: 1)$ & 0.547 & 7.317E-0 \\
\hline
\end{tabular}




\begin{tabular}{|c|c|c|c|}
\hline HMDB11545 & $\operatorname{MG}(20: 3)$ & 0.652 & 3.109E-04 \\
\hline HMDB11549 & $\operatorname{MG}(20: 4)$ & 0.641 & $7.683 \mathrm{E}-05$ \\
\hline HMDB11610 & 5-Taurinomethyl-2-thiouridine & 0.497 & $1.062 \mathrm{E}-03$ \\
\hline HMDB 11670 & 8-Oxo-dGMP & 2.028 & $2.836 \mathrm{E}-07$ \\
\hline HMDB12177 & 5-Methyltetrahydropteroyltri-L-glutamate & 0.475 & $1.676 \mathrm{E}-02$ \\
\hline HMDB12289 & Tetrahydrodipicolinate & 0.041 & $1.030 \mathrm{E}-04$ \\
\hline HMDB12420 & $\operatorname{PS}(20: 3 / 16: 0)$ & 0.261 & $8.257 \mathrm{E}-04$ \\
\hline HMDB12634 & 20-COOH-leukotriene E4 & 0.375 & $3.285 \mathrm{E}-06$ \\
\hline HMDB13034 & Palmitoylglycine & 0.495 & $8.670 \mathrm{E}-03$ \\
\hline HMDB13207 & 9-Hexadecenoylcarnitine & 0.131 & $6.596 \mathrm{E}-04$ \\
\hline HMDB13220 & Beta-Citryl-L-glutamic acid & 0.409 & $1.335 \mathrm{E}-06$ \\
\hline HMDB 13250 & Myristoylglycine & 0.615 & $6.142 \mathrm{E}-04$ \\
\hline HMDB13303 & Pristanoylglycine & 0.394 & $1.260 \mathrm{E}-05$ \\
\hline HMDB13326 & 2-Hydroxylauroylcarnitine & 0.325 & $1.100 \mathrm{E}-04$ \\
\hline HMDB13330 & 3-Hydroxy-cis-5-tetradecenoylcarnitine & 0.527 & $3.409 \mathrm{E}-07$ \\
\hline HMDB13331 & 3, 5-Tetradecadiencarnitine & 0.432 & $7.322 \mathrm{E}-04$ \\
\hline HMDB13334 & 9,12-Hexadecadienoylcarnitine & 0.263 & $3.042 \mathrm{E}-03$ \\
\hline HMDB13335 & 3-Hydroxyhexadecadienoylcarnitine & 0.240 & $1.082 \mathrm{E}-03$ \\
\hline HMDB13631 & Oleoyl glycine & 0.044 & $2.812 \mathrm{E}-04$ \\
\hline HMDB13636 & Pyrroloquinoline quinone & 0.252 & $4.025 \mathrm{E}-07$ \\
\hline HMDB28757 & Aspartyl-Leucine & 0.619 & $3.102 \mathrm{E}-09$ \\
\hline HMDB28789 & Cysteinyl-Gamma-glutamate & 0.277 & $3.065 \mathrm{E}-04$ \\
\hline HMDB28934 & Leucyl-Lysine & 0.156 & $4.021 \mathrm{E}-03$ \\
\hline HMDB29006 & Phenylalanyl-Tryptophan & 0.429 & $1.182 \mathrm{E}-08$ \\
\hline HMDB29110 & Tyrosyl-Lysine & 0.583 & $1.693 \mathrm{E}-04$ \\
\hline HMDB42660 & TG(14:0/20:4/18:0) & 0.220 & $1.530 \mathrm{E}-09$ \\
\hline HMDB42720 & $\mathrm{TG}(14: 0 / 22: 4 / 18: 0)$ & 3.975 & $1.275 \mathrm{E}-03$ \\
\hline HMDB43684 & TG(15:0/18:4/18:0) & 0.123 & $3.670 \mathrm{E}-06$ \\
\hline HMDB59601 & Indan-1-ol & 2.036 & $7.540 \mathrm{E}-06$ \\
\hline HMDB59602 & Indanone & 2.086 & $3.362 \mathrm{E}-06$ \\
\hline HMDB59754 & 3-Methylazelaic acid & 2.699 & $8.585 \mathrm{E}-05$ \\
\hline HMDB60059 & $\mathrm{CE}(17: 0)$ & 0.242 & $3.332 \mathrm{E}-03$ \\
\hline HMDB60274 & Sedoheptulose 1,7-bisphosphate & 4.032 & $1.742 \mathrm{E}-07$ \\
\hline HMDB60343 & 2-(S-Glutathionyl)acetyl glutathione & 0.227 & $1.418 \mathrm{E}-04$ \\
\hline HMDB60812 & cyclic N-Acetylserotonin glucuronide & 0.512 & $2.812 \mathrm{E}-07$ \\
\hline HMDB61661 & 9-hydroxyoctadecanoic acid & 1.501 & $1.724 \mathrm{E}-04$ \\
\hline HMDB61690 & 1-Arachidonoylglycerophosphoinositol & 0.574 & $5.228 \mathrm{E}-08$ \\
\hline HMDB61694 & 1-Oleoylglycerophosphoserine & 0.460 & 4.227E-05 \\
\hline HMDB61698 & 1-Stearoylglycerophosphoserine & 0.569 & $5.187 \mathrm{E}-06$ \\
\hline
\end{tabular}




\begin{tabular}{|c|c|c|c|}
\hline \multicolumn{4}{|c|}{$\begin{array}{l}\text { Supplementary Table 2. Significantly altered metabolites in BEAS-2B cells } \\
\text { treated with high-dose } \mathrm{SiO}_{2} \text { NPs. }\end{array}$} \\
\hline ID & Description & Ratio & q value \\
\hline HMDB00034 & Adenine & 0.264 & $1.814 \mathrm{E}-05$ \\
\hline HMDB00039 & Butyric acid & 4.649 & $1.117 \mathrm{E}-04$ \\
\hline HMDB00050 & Adenosine & 0.326 & 4.059E-06 \\
\hline HMDB00114 & Glycerylphosphorylethanolamine & 0.375 & $1.978 \mathrm{E}-06$ \\
\hline HMDB00177 & L-Histidine & 0.304 & $3.089 \mathrm{E}-07$ \\
\hline HMDB00195 & Inosine & 0.160 & $1.403 \mathrm{E}-05$ \\
\hline HMDB00205 & Phenylpyruvic acid & 1.817 & $2.748 \mathrm{E}-06$ \\
\hline HMDB00244 & Riboflavin & 1.341 & $3.074 \mathrm{E}-03$ \\
\hline HMDB00252 & Sphingosine & 8.583 & 2.677E-08 \\
\hline HMDB00263 & Phosphoenolpyruvic acid & 5.311 & $1.656 \mathrm{E}-02$ \\
\hline HMDB00277 & Sphingosine 1-phosphate & 2.105 & 8.193E-04 \\
\hline HMDB00280 & Phosphoribosyl pyrophosphate & 1.764 & $2.055 \mathrm{E}-03$ \\
\hline HMDB00286 & Uridine diphosphate glucose & 0.150 & 2.337E-09 \\
\hline HMDB00290 & Uridine diphosphate-N-acetylglucosamine & 0.578 & $7.828 \mathrm{E}-07$ \\
\hline HMDB00295 & Uridine 5'-diphosphate & 1.515 & 2.794E-02 \\
\hline HMDB00656 & Cysteineglutathione disulfide & 1.711 & 7.703E-04 \\
\hline HMDB00701 & Hexanoylglycine & 1.294 & 4.627E-03 \\
\hline HMDB00779 & Phenyllactic acid & 0.027 & $4.831 \mathrm{E}-12$ \\
\hline HMDB00806 & Myristic acid & 1.558 & $1.760 \mathrm{E}-02$ \\
\hline HMDB00825 & 3'-Sialyllactose/6'-Sialyllactose & 0.114 & 8.303E-07 \\
\hline HMDB00845 & Neopterin & 1.799 & $3.841 \mathrm{E}-02$ \\
\hline HMDB00935 & Uridine diphosphate glucuronic acid & 0.200 & $2.972 \mathrm{E}-07$ \\
\hline HMDB00939 & S-Adenosylhomocysteine & 3.397 & $6.087 \mathrm{E}-04$ \\
\hline HMDB00942 & Tetrahydroneopterin & 0.142 & $1.090 \mathrm{E}-04$ \\
\hline HMDB00943 & Hypoxanthine & 0.146 & $1.368 \mathrm{E}-02$ \\
\hline HMDB01043 & Arachidonic acid & 2.251 & $8.427 \mathrm{E}-06$ \\
\hline HMDB01049 & Gamma-Glutamylcysteine & 0.295 & $3.568 \mathrm{E}-08$ \\
\hline HMDB01095 & GDP-L-fucose & 0.676 & $1.719 \mathrm{E}-04$ \\
\hline HMDB01125 & Inositol cyclic phosphate & 0.650 & $1.328 \mathrm{E}-05$ \\
\hline HMDB01142 & FMNH2 & 0.443 & $1.968 \mathrm{E}-04$ \\
\hline HMDB01173 & 5'-Methylthioadenosine & 0.254 & $6.784 \mathrm{E}-11$ \\
\hline HMDB01373 & Dephospho-CoA & 1.803 & $2.840 \mathrm{E}-02$ \\
\hline HMDB01476 & 3-Hydroxyanthranilic acid & 2.477 & 4.637E-02 \\
\hline HMDB01520 & Flavin Mononucleotide & 3.341 & $1.941 \mathrm{E}-03$ \\
\hline HMDB01533 & 5,10-Methylene-THF & 0.016 & $8.116 \mathrm{E}-07$ \\
\hline HMDB01570 & Thymidine 3',5'-cyclic monophosphate & 1.580 & $9.772 \mathrm{E}-04$ \\
\hline HMDB01846 & Tetrahydrofolic acid & 0.061 & $1.915 \mathrm{E}-04$ \\
\hline HMDB01976 & Docosapentaenoic acid (22n-6) & 2.728 & $1.806 \mathrm{E}-06$ \\
\hline HMDB02012 & Ubiquinone-1 & 0.097 & $4.085 \mathrm{E}-09$ \\
\hline HMDB02030 & Fructosamine & 2.021 & 8.959E-05 \\
\hline HMDB02095 & Malonylcarnitine & 0.044 & $2.972 \mathrm{E}-05$ \\
\hline
\end{tabular}




\begin{tabular}{|c|c|c|c|}
\hline HMDB02183 & Docosahexaenoic acid & 1.712 & $3.164 \mathrm{E}-03$ \\
\hline HMDB02189 & N8-Acetylspermidine & 0.598 & $1.229 \mathrm{E}-02$ \\
\hline HMDB02206 & Molybdopterin & 3.260 & 3.887E-02 \\
\hline HMDB02224 & 5-Methyldeoxycytidine & 4.174 & $1.150 \mathrm{E}-04$ \\
\hline HMDB02334 & Benzoquinoneacetic acid & 2.431 & $1.656 \mathrm{E}-02$ \\
\hline HMDB02664 & Prostaglandin E2 & 2.157 & $7.825 \mathrm{E}-03$ \\
\hline HMDB02823 & Docosatrienoic acid & 3.008 & 4.564E-04 \\
\hline HMDB02984 & 11-Hydroxyandrosterone & 0.074 & $2.876 \mathrm{E}-09$ \\
\hline HMDB03229 & Palmitoleic acid & 2.150 & $4.564 \mathrm{E}-04$ \\
\hline HMDB03231 & Vaccenic acid & 1.895 & $7.058 \mathrm{E}-06$ \\
\hline HMDB03536 & dIDP & 0.286 & $8.175 \mathrm{E}-06$ \\
\hline HMDB03577 & VPGPR Enterostatin & 1.938 & $6.499 \mathrm{E}-05$ \\
\hline HMDB03601 & (S)-Reticuline & 4.081 & $8.158 \mathrm{E}-08$ \\
\hline HMDB03752 & LysoPC(10:0) & 8.145 & $8.405 \mathrm{E}-04$ \\
\hline HMDB04231 & Pantothenol & 0.079 & $2.726 \mathrm{E}-08$ \\
\hline HMDB04309 & Triterpenoid & 3.114 & $1.169 \mathrm{E}-06$ \\
\hline HMDB04866 & Lactosylceramide (d18:1/12:0) & 0.025 & $1.161 \mathrm{E}-05$ \\
\hline HMDB04976 & Glucosylceramide (d18:1/26:1(17Z)) & 0.090 & $1.714 \mathrm{E}-03$ \\
\hline HMDB04977 & Glucosylceramide (d18:1/26:0) & 0.747 & $1.197 \mathrm{E}-03$ \\
\hline HMDB05060 & Eicosadienoic acid & 2.479 & $3.346 \mathrm{E}-05$ \\
\hline HMDB06236 & Phenylacetaldehyde & 3.413 & $2.615 \mathrm{E}-09$ \\
\hline HMDB06472 & Calcitroic acid & 1.928 & $1.556 \mathrm{E}-03$ \\
\hline HMDB06730 & QYNAD & 0.578 & $2.392 \mathrm{E}-02$ \\
\hline HMDB06752 & Dihydroceramide & 8.575 & $1.740 \mathrm{E}-06$ \\
\hline HMDB07877 & $\mathrm{PC}(14: 0 / 18: 4)$ & 0.057 & 5.937E-06 \\
\hline HMDB08156 & $\mathrm{PC}(18: 2 / 22: 6)$ & 0.068 & $3.308 \mathrm{E}-04$ \\
\hline HMDB08457 & $\mathrm{PC}(20: 4 / \mathrm{P}-18: 1))$ & 3.088 & 7.672E-07 \\
\hline HMDB08786 & $\mathrm{PC}(24: 0 / \mathrm{P}-18: 1)$ & 0.468 & $4.037 \mathrm{E}-03$ \\
\hline HMDB09144 & $\operatorname{PE}(18: 3 / 22: 6)$ & 0.351 & 4.734E-04 \\
\hline HMDB09542 & $\operatorname{PE}(22: 1 / 24: 1)$ & 0.175 & $2.303 \mathrm{E}-04$ \\
\hline HMDB09603 & $\operatorname{PE}(22: 4 / 22: 5)$ & 6.773 & $3.664 \mathrm{E}-13$ \\
\hline HMDB10355 & Cholestane-3,7,12,25-tetrol-3-glucuronide & 0.723 & $9.130 \mathrm{E}-03$ \\
\hline HMDB10378 & 5,8,11-Eicosatrienoic acid & 2.387 & $1.297 \mathrm{E}-07$ \\
\hline HMDB10380 & LysoPC $(14: 1))$ & 3.947 & $8.456 \mathrm{E}-06$ \\
\hline HMDB10387 & LysoPC(18:3) & 0.502 & $7.753 \mathrm{E}-07$ \\
\hline HMDB10399 & LysoPC(22:1) & 5.175 & $3.916 \mathrm{E}-09$ \\
\hline HMDB10405 & LysoPC(24:0) & 1.934 & $3.504 \mathrm{E}-03$ \\
\hline HMDB10674 & PG(18:3/22:6) & 2.608 & $2.863 \mathrm{E}-05$ \\
\hline HMDB10733 & 3-Oxohexadecanoic acid & 1.689 & $6.559 \mathrm{E}-06$ \\
\hline HMDB11177 & L-phenylalanyl-L-proline & 0.126 & $1.645 \mathrm{E}-05$ \\
\hline HMDB11470 & LysoPE(14:0) & 0.683 & $9.015 \mathrm{E}-05$ \\
\hline HMDB11471 & LysoPE(14:1) & 0.689 & $1.646 \mathrm{E}-02$ \\
\hline HMDB11473 & LysoPE(16:0) & 0.760 & $9.235 \mathrm{E}-\mathrm{C}$ \\
\hline HMDB11474 & LysoPE(16:1) & 0.674 & $6.012 \mathrm{E}-0$ \\
\hline
\end{tabular}




\begin{tabular}{|c|c|c|c|}
\hline HMDB11477 & LysoPE(18:2) & 0.746 & $2.504 \mathrm{E}-05$ \\
\hline HMDB11484 & LysoPE(20:3) & 0.772 & $6.074 \mathrm{E}-03$ \\
\hline HMDB11487 & LysoPE(20:4) & 0.786 & 4.143E-04 \\
\hline HMDB11489 & LysoPE(20:5) & 0.766 & $3.375 \mathrm{E}-04$ \\
\hline HMDB11541 & MG(18:4) & 2.089 & $1.200 \mathrm{E}-06$ \\
\hline HMDB11610 & 5-Taurinomethyl-2-thiouridine & 1.572 & $1.314 \mathrm{E}-07$ \\
\hline HMDB11627 & Farnesylcysteine & 0.630 & $1.748 \mathrm{E}-07$ \\
\hline HMDB11684 & $\mathrm{N}(6)-($ Octanoyl)lysine & 0.090 & $3.826 \mathrm{E}-04$ \\
\hline HMDB11741 & Gamma-Glutamyltyrosine & 1.348 & $5.070 \mathrm{E}-05$ \\
\hline HMDB11760 & $\operatorname{Cer}(\mathrm{d} 18: 0 / 16: 0)$ & 3.059 & 2.191E-03 \\
\hline HMDB12082 & LysoSM(d18:0) & 0.179 & $3.224 \mathrm{E}-11$ \\
\hline HMDB12932 & Dynorphin A (6-8) & 0.651 & $2.480 \mathrm{E}-04$ \\
\hline HMDB13250 & Myristoylglycine & 2.202 & $2.818 \mathrm{E}-02$ \\
\hline HMDB13286 & N-Undecanoylglycine & 0.706 & $3.161 \mathrm{E}-07$ \\
\hline HMDB13303 & Pristanoylglycine & 0.463 & $2.295 \mathrm{E}-07$ \\
\hline HMDB13317 & Tridecanoylglycine & 7.774 & $2.461 \mathrm{E}-05$ \\
\hline HMDB13330 & 3-Hydroxy-cis-5-tetradecenoylcarnitine & 0.595 & $1.784 \mathrm{E}-06$ \\
\hline HMDB13331 & 3, 5-Tetradecadiencarnitine & 0.270 & $1.734 \mathrm{E}-04$ \\
\hline HMDB28789 & Cysteinyl-Gamma-glutamate & 2.634 & $2.316 \mathrm{E}-03$ \\
\hline HMDB28829 & Glutamyl-Threonine & 5.775 & $3.586 \mathrm{E}-11$ \\
\hline HMDB28950 & Lysyl-Glutamate & 1.849 & $5.045 \mathrm{E}-04$ \\
\hline HMDB28979 & Methionyl-Methionine & 1.377 & $9.090 \mathrm{E}-06$ \\
\hline HMDB28985 & Methionyl-Tyrosine & 1.806 & $3.877 \mathrm{E}-03$ \\
\hline HMDB29007 & Phenylalanyl-Tyrosine & 1.974 & 3.417E-06 \\
\hline HMDB29087 & Tryptophyl-Leucine & 0.028 & $6.074 \mathrm{E}-03$ \\
\hline HMDB29097 & Tryptophyl-Gamma-glutamate & 0.397 & $3.430 \mathrm{E}-02$ \\
\hline HMDB38057 & Dehydrophytosphingosine & 2.267 & $8.186 \mathrm{E}-05$ \\
\hline HMDB42036 & Thymidine glycol & 0.617 & $4.320 \mathrm{E}-03$ \\
\hline HMDB42660 & TG(14:0/20:4/18:0) & 0.018 & $2.331 \mathrm{E}-05$ \\
\hline HMDB42720 & TG(14:0/22:4/18:0) & 2.475 & $1.958 \mathrm{E}-02$ \\
\hline HMDB43684 & TG(15:0/18:4/18:0) & 0.060 & $1.375 \mathrm{E}-06$ \\
\hline HMDB59571 & Oxychlordane & 6.321 & $2.576 \mathrm{E}-09$ \\
\hline HMDB59614 & Riboflavin cyclic-4',5'-phosphate & 3.706 & $1.978 \mathrm{E}-06$ \\
\hline HMDB59645 & beta-nicotinamide D-ribonucleotide & 2.131 & 5.013E-03 \\
\hline HMDB59729 & 3,4-Methylenesebacic acid & 2.497 & $1.681 \mathrm{E}-07$ \\
\hline HMDB59744 & 3,4-Methyleneazelaic acid & 0.022 & $1.331 \mathrm{E}-06$ \\
\hline HMDB60129 & 24-Oxo-1alpha,23,25-trihydroxyvitamin D3 & 3.854 & $6.784 \mathrm{E}-11$ \\
\hline HMDB60179 & 3-Sulfinato-L-alaninate & 1.661 & $2.002 \mathrm{E}-05$ \\
\hline HMDB60830 & Melatonin glucuronide & 1.433 & $2.215 \mathrm{E}-02$ \\
\hline HMDB61522 & PE(MonoMe(11,3)/MonoMe(11,5)) & 2.375 & $3.871 \mathrm{E}-02$ \\
\hline HMDB61641 & 3-hydroxypentadecanoyl carnitine & 1.914 & $3.438 \mathrm{E}-05$ \\
\hline HMDB61658 & 3-hydroxyhexadecanoic acid & 1.722 & $1.205 \mathrm{E}-07$ \\
\hline HMDB61692 & $\begin{array}{l}\text { 1-Linoleoylglycerophosphocholine/ } \\
\text { 2-Linoleoylglycerophosphocholine }\end{array}$ & 5.477 & $5.057 \mathrm{E}-10$ \\
\hline
\end{tabular}




\begin{tabular}{|c|c|c|c|}
\hline \multicolumn{4}{|c|}{$\begin{array}{l}\text { Supplementary Table 3. Significantly altered metabolites in BEAS-2B cells } \\
\text { treated with high-dose } \mathrm{TiO}_{2} \text { NPs. }\end{array}$} \\
\hline ID & Description & Ratio & q value \\
\hline HMDB00039 & Butyric acid & 7.275 & $1.803 \mathrm{E}-05$ \\
\hline HMDB00052 & Argininosuccinic acid & 8.597 & $4.320 \mathrm{E}-08$ \\
\hline HMDB00070 & Pipecolic acid & 2.062 & $9.011 \mathrm{E}-03$ \\
\hline HMDB00099 & L-Cystathionine & 2.242 & $1.129 \mathrm{E}-03$ \\
\hline HMDB00114 & Glycerylphosphorylethanolamine & 0.409 & $3.054 \mathrm{E}-07$ \\
\hline HMDB00125 & Glutathione & 0.554 & $3.450 \mathrm{E}-04$ \\
\hline HMDB00201 & L-Acetylcarnitine & 0.405 & $3.942 \mathrm{E}-09$ \\
\hline HMDB00205 & Phenylpyruvic acid & 2.256 & $2.792 \mathrm{E}-05$ \\
\hline HMDB00230 & N-Acetylneuraminic acid & 1.570 & $3.052 \mathrm{E}-06$ \\
\hline HMDB00244 & Riboflavin & 1.648 & $1.481 \mathrm{E}-04$ \\
\hline HMDB00252 & Sphingosine & 6.715 & $5.286 \mathrm{E}-08$ \\
\hline HMDB00263 & Phosphoenolpyruvic acid & 3.378 & $3.696 \mathrm{E}-02$ \\
\hline HMDB00265 & Liothyronine & 0.661 & $1.337 \mathrm{E}-06$ \\
\hline HMDB00272 & Phosphoserine & 0.617 & $2.312 \mathrm{E}-03$ \\
\hline HMDB00277 & Sphingosine 1-phosphate & 1.515 & $7.549 \mathrm{E}-04$ \\
\hline HMDB00286 & Uridine diphosphate glucose & 0.137 & $2.408 \mathrm{E}-09$ \\
\hline HMDB00290 & Uridine diphosphate-N-acetylglucosamine & 0.536 & $1.033 \mathrm{E}-06$ \\
\hline HMDB00301 & Urocanic acid & 2.615 & $3.495 \mathrm{E}-06$ \\
\hline HMDB00623 & Dodecanedioic acid & 1.295 & $2.809 \mathrm{E}-04$ \\
\hline HMDB00672 & Hexadecanedioic acid & 1.598 & $4.751 \mathrm{E}-03$ \\
\hline HMDB00712 & $\begin{array}{l}\text { Hexadecanedioic acid mono-L-carnitine } \\
\text { ester }\end{array}$ & 1.924 & $8.223 \mathrm{E}-05$ \\
\hline HMDB00779 & Phenyllactic acid & 0.028 & $3.856 \mathrm{E}-11$ \\
\hline HMDB00806 & Myristic acid & 1.769 & $5.042 \mathrm{E}-03$ \\
\hline HMDB00825 & 3'-Sialyllactose/6'-Sialyllactose & 0.195 & $3.952 \mathrm{E}-08$ \\
\hline HMDB00845 & Neopterin & 2.025 & $2.222 \mathrm{E}-02$ \\
\hline HMDB00872 & Tetradecanedioic acid & 1.519 & $4.515 \mathrm{E}-04$ \\
\hline HMDB00888 & Undecanedioic acid & 1.266 & $1.206 \mathrm{E}-03$ \\
\hline HMDB00905 & Deoxyadenosine monophosphate & 0.558 & $2.671 \mathrm{E}-02$ \\
\hline HMDB00935 & Uridine diphosphate glucuronic acid & 0.150 & $5.829 \mathrm{E}-06$ \\
\hline HMDB00939 & S-Adenosylhomocysteine & 1.786 & $1.945 \mathrm{E}-02$ \\
\hline HMDB00942 & Tetrahydroneopterin & 0.159 & $1.189 \mathrm{E}-04$ \\
\hline HMDB00943 & Threonic acid & 2.376 & $4.665 \mathrm{E}-05$ \\
\hline HMDB00962 & Lipoamide & 2.209 & $5.012 \mathrm{E}-03$ \\
\hline HMDB01043 & Arachidonic acid & 2.767 & $1.414 \mathrm{E}-05$ \\
\hline HMDB01095 & GDP-L-fucose & 0.692 & $1.691 \mathrm{E}-03$ \\
\hline HMDB01173 & 5'-Methylthioadenosine & 0.497 & $4.966 \mathrm{E}-07$ \\
\hline HMDB01272 & Nicotine glucuronide & 1.481 & $2.773 \mathrm{E}-06$ \\
\hline HMDB01278 & Presqualene diphosphate & 6.428 & $5.878 \mathrm{E}-08$ \\
\hline HMDB01280 & 2-Aminomuconic acid semialdehyde & 1.741 & $1.851 \mathrm{E}-05$ \\
\hline HMDB01373 & Dephospho-CoA & 1.811 & $2.596 \mathrm{E}-02$ \\
\hline
\end{tabular}




\begin{tabular}{|c|c|c|c|}
\hline HMDB01520 & Flavin Mononucleotide & 4.442 & 4.840E-04 \\
\hline HMDB01533 & 5,10-Methylene-THF & 0.030 & $6.182 \mathrm{E}-06$ \\
\hline HMDB01846 & Tetrahydrofolic acid & 0.094 & $3.326 \mathrm{E}-04$ \\
\hline HMDB01976 & Docosapentaenoic acid (22n-6) & 3.134 & 4.992E-07 \\
\hline HMDB02012 & Ubiquinone-1 & 0.095 & $6.937 \mathrm{E}-11$ \\
\hline HMDB02030 & Fructosamine & 4.788 & $6.481 \mathrm{E}-08$ \\
\hline HMDB02053 & Histidylproline diketopiperazine & 1.502 & $1.144 \mathrm{E}-03$ \\
\hline HMDB02088 & N-Oleoylethanolamine & 0.639 & $3.520 \mathrm{E}-02$ \\
\hline HMDB02183 & Docosahexaenoic acid & 2.005 & 7.935E-04 \\
\hline HMDB02224 & 5-Methyldeoxycytidine & 8.124 & $2.957 \mathrm{E}-05$ \\
\hline HMDB02327 & 1,11-Undecanedicarboxylic acid & 1.344 & $1.953 \mathrm{E}-05$ \\
\hline HMDB02334 & Benzoquinoneacetic acid & 7.787 & 8.282E-04 \\
\hline HMDB02343 & 5,6-DHET & 2.667 & 5.373E-13 \\
\hline HMDB02664 & Prostaglandin E2 & 2.063 & $1.085 \mathrm{E}-03$ \\
\hline HMDB02757 & Cysteic acid & 2.651 & $6.353 \mathrm{E}-04$ \\
\hline HMDB02823 & Docosatrienoic acid & 2.887 & $5.078 \mathrm{E}-04$ \\
\hline HMDB02984 & 11-Hydroxyandrosterone & 0.082 & $5.685 \mathrm{E}-09$ \\
\hline HMDB03229 & Palmitoleic acid & 3.356 & $3.414 \mathrm{E}-05$ \\
\hline HMDB03231 & Vaccenic acid & 2.132 & $1.506 \mathrm{E}-06$ \\
\hline HMDB03577 & VPGPR Enterostatin & 2.288 & $1.008 \mathrm{E}-05$ \\
\hline HMDB03601 & (S)-Reticuline & 9.880 & $1.808 \mathrm{E}-08$ \\
\hline HMDB03752 & LysoPC(10:0) & 6.495 & $2.965 \mathrm{E}-04$ \\
\hline HMDB03892 & (S)-N-Methylsalsolinol & 3.013 & $2.056 \mathrm{E}-04$ \\
\hline HMDB04231 & Pantothenol & 0.084 & $5.642 \mathrm{E}-09$ \\
\hline \multirow[t]{2}{*}{ HMDB04326 } & 2'-O-Methyladenosine/ & 1.535 & $9.470 \mathrm{E}-04$ \\
\hline & 3'-O-Methyladenosine & & \\
\hline HMDB04645 & S-Nitrosoglutathione & 0.743 & $1.613 \mathrm{E}-03$ \\
\hline HMDB04866 & Lactosylceramide (d18:1/12:0) & 0.075 & $1.812 \mathrm{E}-07$ \\
\hline HMDB04976 & Glucosylceramide (d18:1/26:1(17Z)) & 0.183 & $1.820 \mathrm{E}-03$ \\
\hline HMDB05060 & Eicosadienoic acid & 2.696 & $6.987 \mathrm{E}-05$ \\
\hline HMDB06236 & Phenylacetaldehyde & 6.135 & $1.701 \mathrm{E}-11$ \\
\hline HMDB06353 & Dolichol phosphate & 2.007 & $7.209 \mathrm{E}-03$ \\
\hline HMDB06472 & Calcitroic acid & 1.971 & $1.412 \mathrm{E}-03$ \\
\hline HMDB06587 & Lactodifucotetraose & 3.006 & $1.667 \mathrm{E}-03$ \\
\hline HMDB06752 & Dihydroceramide & 7.695 & $6.006 \mathrm{E}-06$ \\
\hline HMDB07877 & $\operatorname{PC}(14: 0 / 18: 4)$ & 0.255 & $2.187 \mathrm{E}-05$ \\
\hline HMDB08156 & $\operatorname{PC}(18: 2 / 22: 6)$ & 0.241 & $7.182 \mathrm{E}-05$ \\
\hline HMDB08210 & $\operatorname{PC}(18: 3 / 20: 2)$ & 1.782 & $4.085 \mathrm{E}-04$ \\
\hline HMDB08387 & $\operatorname{PC}(20: 3 / 22: 6)$ & 1.891 & $3.559 \mathrm{E}-08$ \\
\hline HMDB08457 & $\mathrm{PC}(20: 4 / \mathrm{P}-18: 1)$ & 3.049 & $1.130 \mathrm{E}-05$ \\
\hline HMDB08649 & $\operatorname{PC}(22: 4 / 22: 6)$ & 2.017 & $1.570 \mathrm{E}-06$ \\
\hline HMDB08821 & $\operatorname{PE}(14: 0 / 14: 0)$ & 1.974 & $2.857 \mathrm{E}-10$ \\
\hline HMDB08834 & $\operatorname{PE}(14: 0 / 20: 1)$ & 3.471 & $3.212 \mathrm{E}-08$ \\
\hline HMDB09603 & $\operatorname{PE}(22: 4 / 22: 5)$ & 11.618 & $9.212 \mathrm{E}-13$ \\
\hline
\end{tabular}




\begin{tabular}{|c|c|c|c|}
\hline HMDB09819 & $\operatorname{PI}(18: 0 / 22: 5)$ & 4.814 & $4.436 \mathrm{E}-02$ \\
\hline HMDB10331 & Palmitoyl glucuronide & 3.339 & $3.677 \mathrm{E}-06$ \\
\hline HMDB10337 & 6-Dehydrotestosterone glucuronide & 18.383 & 4.963E-06 \\
\hline HMDB10355 & Cholestane-3,7,12,25-tetrol-3-glucuronide & 0.586 & $1.378 \mathrm{E}-03$ \\
\hline HMDB10364 & 11-Hydroxyprogesterone 11-glucuronide & 37.725 & $3.263 \mathrm{E}-06$ \\
\hline HMDB 10378 & 5,8,11-Eicosatrienoic acid & 2.351 & $3.457 \mathrm{E}-07$ \\
\hline HMDB10399 & LysoPC(22:1) & 8.411 & 5.610E-09 \\
\hline HMDB10404 & LysoPC(22:6) & 1.838 & $9.821 \mathrm{E}-07$ \\
\hline HMDB10674 & $\operatorname{PG}(18: 3 / 22: 6)$ & 17.289 & $2.034 \mathrm{E}-07$ \\
\hline HMDB10733 & 3-Oxohexadecanoic acid & 3.200 & $2.525 \mathrm{E}-06$ \\
\hline HMDB11164 & L-beta-aspartyl-L-glutamic acid & 1.489 & $5.277 \mathrm{E}-03$ \\
\hline HMDB11171 & L-gamma-glutamyl-L-leucine & 0.639 & $4.536 \mathrm{E}-05$ \\
\hline HMDB11177 & L-phenylalanyl-L-proline & 0.162 & $5.321 \mathrm{E}-06$ \\
\hline HMDB 11470 & LysoPE(14:0) & 0.624 & $6.799 \mathrm{E}-05$ \\
\hline HMDB11473 & LysoPE(16:0) & 7.769 & $5.384 \mathrm{E}-10$ \\
\hline HMDB11474 & LysoPE(16:1) & 0.667 & $2.138 \mathrm{E}-07$ \\
\hline HMDB11477 & LysoPE(18:2) & 0.737 & $1.907 \mathrm{E}-05$ \\
\hline HMDB 11478 & LysoPE(18:3) & 0.695 & $3.414 \mathrm{E}-05$ \\
\hline HMDB11484 & LysoPE(20:3) & 0.682 & $7.058 \mathrm{E}-04$ \\
\hline HMDB11487 & LysoPE(20:4) & 0.710 & $3.737 \mathrm{E}-05$ \\
\hline HMDB11489 & LysoPE(20:5) & 0.683 & $1.962 \mathrm{E}-05$ \\
\hline HMDB 11513 & LysoPE(20:2) & 0.730 & $1.957 \mathrm{E}-02$ \\
\hline HMDB11526 & LysoPE(22:6) & 0.772 & $3.412 \mathrm{E}-05$ \\
\hline HMDB11532 & $\operatorname{MG}(15: 0)$ & 1.459 & $5.449 \mathrm{E}-03$ \\
\hline HMDB11541 & $\operatorname{MG}(18: 4)$ & 2.224 & $2.679 \mathrm{E}-05$ \\
\hline HMDB11545 & $\operatorname{MG}(20: 3)$ & 0.619 & $2.964 \mathrm{E}-03$ \\
\hline HMDB11610 & 5-Taurinomethyl-2-thiouridine & 1.493 & $1.242 \mathrm{E}-02$ \\
\hline HMDB11627 & Farnesylcysteine & 0.646 & $2.682 \mathrm{E}-07$ \\
\hline HMDB11684 & $\mathrm{N}(6)-($ Octanoyl)lysine & 0.046 & 4.612E-04 \\
\hline HMDB11687 & Phenylbutyrylglutamine & 6.362 & $1.856 \mathrm{E}-08$ \\
\hline HMDB 11720 & UDP-N-acetylmuraminate & 2.457 & $1.290 \mathrm{E}-02$ \\
\hline HMDB11739 & Galactinol dihydrate & 1.499 & $2.463 \mathrm{E}-02$ \\
\hline HMDB12082 & LysoSM(d18:0) & 0.221 & $3.133 \mathrm{E}-06$ \\
\hline HMDB12154 & 3-Dehydrocarnitine & 1.351 & $5.449 \mathrm{E}-03$ \\
\hline HMDB12305 & UDP-L-rhamnose & 1.746 & $9.160 \mathrm{E}-03$ \\
\hline HMDB13238 & Heptanoylcarnitine & 1.752 & $6.230 \mathrm{E}-07$ \\
\hline HMDB13250 & Myristoylglycine & 2.014 & $3.477 \mathrm{E}-02$ \\
\hline HMDB13303 & Pristanoylglycine & 0.473 & $5.616 \mathrm{E}-05$ \\
\hline HMDB13317 & Tridecanoylglycine & 22.039 & $3.604 \mathrm{E}-06$ \\
\hline HMDB13327 & Dodecanedioylcarnitine & 2.658 & $3.322 \mathrm{E}-0$ \\
\hline HMDB13331 & 3,5 -Tetradecadiencarnitine & 0.414 & $7.060 \mathrm{E}-03$ \\
\hline HMDB28684 & Alanyl-Cysteine & 2.264 & $1.457 \mathrm{E}-06$ \\
\hline HMDB28696 & Alanyl-Serine & 2.761 & $4.537 \mathrm{E}-\mathrm{C}$ \\
\hline HMDB28704 & Arginyl-Asparagine & 1.527 & $1.918 \mathrm{E}-0$ \\
\hline
\end{tabular}




\begin{tabular}{llll} 
HMDB28755 & Aspartyl-Histidine & 7.842 & $4.587 \mathrm{E}-03$ \\
HMDB28789 & Cysteinyl-Gamma-glutamate & 6.299 & $1.086 \mathrm{E}-04$ \\
HMDB28799 & Glutaminyl-Histidine & 2.266 & $7.745 \mathrm{E}-05$ \\
HMDB28829 & Glutamyl-Threonine & 5.400 & $6.306 \mathrm{E}-09$ \\
HMDB28831 & Glutamyl-Tyrosine & 2.117 & $9.354 \mathrm{E}-07$ \\
HMDB28893 & Histidinyl-Proline & 3.310 & $2.216 \mathrm{E}-03$ \\
HMDB28950 & Lysyl-Glutamate & 1.998 & $1.969 \mathrm{E}-04$ \\
HMDB28985 & Methionyl-Tyrosine & 1.674 & $6.216 \mathrm{E}-03$ \\
HMDB29007 & Phenylalanyl-Tyrosine & 1.669 & $3.600 \mathrm{E}-05$ \\
HMDB29087 & Tryptophyl-Leucine & 0.045 & $3.230 \mathrm{E}-02$ \\
HMDB29105 & Tyrosyl-Glycine & 0.778 & $6.858 \mathrm{E}-05$ \\
HMDB29110 & Tyrosyl-Lysine & 2.128 & $1.525 \mathrm{E}-05$ \\
HMDB29586 & Octadecylamine & 0.307 & $2.484 \mathrm{E}-05$ \\
HMDB38057 & Dehydrophytosphingosine & 2.072 & $1.577 \mathrm{E}-04$ \\
HMDB42036 & Thymidine glycol & 0.435 & $1.401 \mathrm{E}-02$ \\
HMDB42660 & TG(14:0/20:4/18:0) & 0.070 & $9.664 \mathrm{E}-06$ \\
HMDB43684 & TG(15:0/18:4/18:0) & 0.140 & $3.211 \mathrm{E}-05$ \\
HMDB59571 & Oxychlordane & 14.559 & $2.040 \mathrm{E}-12$ \\
HMDB59614 & Riboflavin cyclic-4',5'-phosphate & 1.871 & $8.371 \mathrm{E}-06$ \\
HMDB59645 & beta-nicotinamide D-ribonucleotide & 2.182 & $9.095 \mathrm{E}-03$ \\
HMDB59717 & Glu-Val & 0.519 & $2.329 \mathrm{E}-03$ \\
HMDB59729 & 3,4-Methylenesebacic acid & 3.101 & $5.530 \mathrm{E}-07$ \\
HMDB59744 & 3,4-Methyleneazelaic acid & 0.025 & $4.986 \mathrm{E}-10$ \\
HMDB60129 & 24-Oxo-1alpha,23,25-trihydroxyvitamin & 6.879 & $5.373 \mathrm{E}-13$ \\
HMDB61522 & D3 & & \\
HMDB61636 & 3-hydroxydecanoyl carnitine & 11.695 & $2.403 \mathrm{E}-04$ \\
HMDB61639 & 3-hydroxytridecanoyl carnitine & 3.279 & $1.568 \mathrm{E}-04$ \\
HMDB61641 & 3-hydroxypentadecanoyl carnitine & 1.866 & $7.529 \mathrm{E}-07$ \\
HMDB61653 & 3-hydroxyheptanoic acid & 3.152 & $8.826 \mathrm{E}-08$ \\
HMDB61692 & 1-Linoleoylglycerophosphocholine/ & 1.731 & $4.585 \mathrm{E}-03$ \\
& 2-Linoleoylglycerophosphocholine & 1.544 & $9.486 \mathrm{E}-07$ \\
& & & \\
\hline
\end{tabular}




\begin{tabular}{|c|c|c|c|}
\hline \multicolumn{4}{|c|}{$\begin{array}{l}\text { Supplementary Table 4. Significantly altered metabolites in BEAS-2B cells } \\
\text { treated with high-dose } \mathrm{CeO}_{2} \text { NPs. }\end{array}$} \\
\hline ID & Description & Ratio & q value \\
\hline HMDB00024 & 3-O-Sulfogalactosylceramide (d18:1/24:0) & 0.384 & $2.306 \mathrm{E}-03$ \\
\hline HMDB00039 & Butyric acid & 0.700 & $3.338 \mathrm{E}-02$ \\
\hline HMDB00062 & L-Carnitine & 1.280 & $3.269 \mathrm{E}-02$ \\
\hline HMDB00099 & L-Cystathionine & 1.841 & 4.747E-03 \\
\hline HMDB00114 & Glycerylphosphorylethanolamine & 0.361 & $1.222 \mathrm{E}-06$ \\
\hline HMDB00158 & L-Tyrosine & 0.716 & $2.169 \mathrm{E}-02$ \\
\hline HMDB00201 & L-Acetylcarnitine & 0.153 & 3.953E-08 \\
\hline HMDB00205 & Phenylpyruvic acid & 2.566 & $1.841 \mathrm{E}-06$ \\
\hline HMDB00244 & Riboflavin & 1.350 & $6.788 \mathrm{E}-03$ \\
\hline HMDB00252 & Sphingosine & 7.547 & $2.080 \mathrm{E}-08$ \\
\hline HMDB00277 & Sphingosine 1-phosphate & 1.721 & $2.336 \mathrm{E}-05$ \\
\hline HMDB00286 & Uridine diphosphate glucose & 0.079 & 3.633E-08 \\
\hline HMDB00290 & Uridine diphosphate-N-acetylglucosamine & 0.598 & $1.158 \mathrm{E}-06$ \\
\hline HMDB00296 & Uridine & 1.877 & $8.708 \mathrm{E}-04$ \\
\hline HMDB00300 & Uracil & 1.892 & $2.701 \mathrm{E}-07$ \\
\hline HMDB00651 & Decanoylcarnitine & 1.989 & 3.727E-05 \\
\hline HMDB00653 & Cholesterol sulfate & 1.738 & $1.871 \mathrm{E}-06$ \\
\hline HMDB00656 & Cysteineglutathione disulfide & 0.534 & 4.244E-04 \\
\hline HMDB00705 & Hexanoylcarnitine & 0.682 & $2.400 \mathrm{E}-02$ \\
\hline HMDB00712 & Hexadecanedioic acid mono-L-carnitine ester & 1.395 & $1.425 \mathrm{E}-04$ \\
\hline HMDB00735 & Hydroxyphenylacetylglycine & 1.623 & $2.563 \mathrm{E}-04$ \\
\hline HMDB00779 & Phenyllactic acid & 0.031 & $5.885 \mathrm{E}-11$ \\
\hline HMDB00806 & Myristic acid & 1.537 & $1.740 \mathrm{E}-02$ \\
\hline HMDB00825 & 3'-Sialyllactose/6'-Sialyllactose & 0.154 & $3.259 \mathrm{E}-05$ \\
\hline HMDB00888 & Undecanedioic acid & 0.402 & $4.578 \mathrm{E}-05$ \\
\hline HMDB00935 & Uridine diphosphate glucuronic acid & 0.087 & $1.296 \mathrm{E}-08$ \\
\hline HMDB00939 & S-Adenosylhomocysteine & 2.303 & $3.081 \mathrm{E}-03$ \\
\hline HMDB00942 & Tetrahydroneopterin & 0.184 & $1.775 \mathrm{E}-07$ \\
\hline HMDB01043 & Arachidonic acid & 2.858 & $2.551 \mathrm{E}-06$ \\
\hline HMDB01095 & GDP-L-fucose & 0.658 & 7.870E-04 \\
\hline HMDB01248 & FAD & 0.334 & $1.083 \mathrm{E}-02$ \\
\hline HMDB01272 & Nicotine glucuronide & 1.358 & $6.236 \mathrm{E}-05$ \\
\hline HMDB01278 & Presqualene diphosphate & 0.251 & $2.822 \mathrm{E}-02$ \\
\hline HMDB01413 & Citicoline & 2.983 & $4.827 \mathrm{E}-05$ \\
\hline HMDB01520 & Flavin Mononucleotide & 7.903 & $2.069 \mathrm{E}-04$ \\
\hline HMDB01533 & 5,10-Methylene-THF & 0.030 & $2.516 \mathrm{E}-06$ \\
\hline HMDB01570 & Thymidine 3',5'-cyclic monophosphate & 1.630 & $4.739 \mathrm{E}-03$ \\
\hline HMDB01846 & Tetrahydrofolic acid & 0.151 & $2.413 \mathrm{E}-05$ \\
\hline HMDB01961 & 1,7-Dimethylguanosine & 1.663 & $6.432 \mathrm{E}-04$ \\
\hline HMDB01976 & Docosapentaenoic acid (22n-6) & 3.309 & $9.079 \mathrm{E}-07$ \\
\hline HMDB02012 & Ubiquinone-1 & 0.096 & $4.607 \mathrm{E}-08$ \\
\hline
\end{tabular}




\begin{tabular}{|c|c|c|c|}
\hline HMDB02014 & cis-5-Tetradecenoylcarnitine & 2.117 & $2.053 \mathrm{E}-08$ \\
\hline HMDB02030 & Fructosamine & 2.326 & $2.089 \mathrm{E}-05$ \\
\hline HMDB02088 & N-Oleoylethanolamine & 1.680 & $3.893 \mathrm{E}-06$ \\
\hline HMDB02135 & S-(3-oxo-3-carboxy-n-propyl)cysteine & 1.814 & $5.435 \mathrm{E}-03$ \\
\hline HMDB02183 & Docosahexaenoic acid & 2.104 & $5.045 \mathrm{E}-04$ \\
\hline HMDB02206 & Molybdopterin & 0.397 & 4.870E-02 \\
\hline HMDB02250 & Dodecanoylcarnitine & 2.180 & 8.347E-07 \\
\hline HMDB02334 & Benzoquinoneacetic acid & 2.752 & $9.199 \mathrm{E}-03$ \\
\hline HMDB02664 & Prostaglandin E2 & 2.637 & $3.085 \mathrm{E}-03$ \\
\hline HMDB02823 & Docosatrienoic acid & 2.411 & $6.669 \mathrm{E}-03$ \\
\hline HMDB02984 & 11-Hydroxyandrosterone & 0.032 & $5.082 \mathrm{E}-08$ \\
\hline HMDB03134 & Biocytin & 0.710 & $4.211 \mathrm{E}-04$ \\
\hline HMDB03229 & Palmitoleic acid & 2.841 & $1.316 \mathrm{E}-04$ \\
\hline HMDB03231 & Vaccenic acid & 1.962 & $9.586 \mathrm{E}-06$ \\
\hline HMDB03447 & Tryptophanol & 2.043 & 5.929E-04 \\
\hline HMDB03752 & LysoPC(10:0) & 7.095 & $1.444 \mathrm{E}-04$ \\
\hline HMDB04047 & Adipoyl-CoA & 0.585 & $1.577 \mathrm{E}-02$ \\
\hline HMDB04231 & Pantothenol & 0.091 & $2.090 \mathrm{E}-07$ \\
\hline HMDB04242 & 11-Dehydro-thromboxane B2 & 2.874 & $7.614 \mathrm{E}-06$ \\
\hline HMDB04326 & $\begin{array}{l}\text { 2'-O-Methyladenosine/ } \\
\text { 3'-O-Methyladenosine }\end{array}$ & 1.360 & 4.802E-03 \\
\hline HMDB04645 & S-Nitrosoglutathione & 0.745 & $1.262 \mathrm{E}-03$ \\
\hline HMDB05060 & Eicosadienoic acid & 2.187 & $2.144 \mathrm{E}-04$ \\
\hline HMDB05066 & Tetradecanoylcarnitine & 2.208 & $1.954 \mathrm{E}-06$ \\
\hline HMDB06049 & O-Phosphotyrosine & 0.550 & $4.288 \mathrm{E}-03$ \\
\hline HMDB06210 & Heptadecanoyl carnitine & 1.338 & $2.355 \mathrm{E}-02$ \\
\hline HMDB06319 & $\begin{array}{l}\text { Alpha-linolenyl carnitine/ } \\
\text { Gamma-linolenyl carnitine }\end{array}$ & 1.961 & $5.148 \mathrm{E}-05$ \\
\hline HMDB06472 & Calcitroic acid & 2.144 & $5.047 \mathrm{E}-04$ \\
\hline HMDB06752 & Dihydroceramide & 6.667 & $5.534 \mathrm{E}-06$ \\
\hline HMDB07850 & $\operatorname{LPA}(18: 0)$ & 1.735 & $5.636 \mathrm{E}-04$ \\
\hline HMDB07851 & LPA(18:1) & 1.517 & $1.461 \mathrm{E}-03$ \\
\hline HMDB07877 & $\mathrm{PC}(14: 0 / 18: 4)$ & 0.165 & $1.010 \mathrm{E}-06$ \\
\hline HMDB08156 & $\operatorname{PC}(18: 2 / 22: 6)$ & 0.149 & $1.081 \mathrm{E}-03$ \\
\hline HMDB09144 & $\operatorname{PE}(18: 3 / 22: 6)$ & 0.256 & $2.215 \mathrm{E}-03$ \\
\hline HMDB10378 & 5,8,11-Eicosatrienoic acid & 2.686 & 7.198E-08 \\
\hline HMDB10387 & LysoPC(18:3) & 1.923 & $3.760 \mathrm{E}-07$ \\
\hline HMDB10397 & LysoPC(20:5) & 1.379 & $1.140 \mathrm{E}-04$ \\
\hline HMDB10733 & 3-Oxohexadecanoic acid & 1.510 & $4.245 \mathrm{E}-06$ \\
\hline HMDB11164 & L-beta-aspartyl-L-glutamic acid & 2.604 & $1.772 \mathrm{E}-08$ \\
\hline HMDB11177 & L-phenylalanyl-L-proline & 0.169 & $1.199 \mathrm{E}-06$ \\
\hline HMDB11470 & LysoPE(14:0) & 0.741 & $8.547 \mathrm{E}-05$ \\
\hline HMDB11471 & LysoPE(14:1) & 0.730 & $1.357 \mathrm{E}-0$ \\
\hline HMDB11473 & LysoPE(16:0) & 0.716 & $2.777 \mathrm{E}-04$ \\
\hline
\end{tabular}




\begin{tabular}{|c|c|c|c|}
\hline HMDB11474 & LysoPE(16:1) & 0.746 & $1.171 \mathrm{E}-05$ \\
\hline HMDB11477 & LysoPE(18:2) & 0.795 & $1.892 \mathrm{E}-04$ \\
\hline HMDB 11478 & LysoPE(18:3) & 0.767 & $2.626 \mathrm{E}-04$ \\
\hline HMDB11484 & LysoPE(20:3) & 0.694 & $1.412 \mathrm{E}-03$ \\
\hline HMDB11487 & LysoPE(20:4) & 0.780 & $2.792 \mathrm{E}-04$ \\
\hline HMDB11489 & LysoPE(20:5) & 0.768 & $2.045 \mathrm{E}-04$ \\
\hline HMDB11513 & LysoPE(20:2) & 0.688 & $5.655 \mathrm{E}-03$ \\
\hline HMDB11535 & $\operatorname{MG}(18: 0)$ & 1.419 & $1.244 \mathrm{E}-06$ \\
\hline HMDB 11538 & $\operatorname{MG}(18: 2)$ & 0.690 & $5.857 \mathrm{E}-04$ \\
\hline HMDB11541 & $\operatorname{MG}(18: 4)$ & 2.113 & $4.633 \mathrm{E}-06$ \\
\hline HMDB11627 & Farnesylcysteine & 0.655 & $7.832 \mathrm{E}-07$ \\
\hline HMDB11687 & Phenylbutyrylglutamine & 2.685 & $1.478 \mathrm{E}-06$ \\
\hline HMDB11720 & UDP-N-acetylmuraminate & 2.549 & $1.051 \mathrm{E}-02$ \\
\hline HMDB11739 & Galactinol dihydrate & 3.257 & $1.278 \mathrm{E}-04$ \\
\hline HMDB12082 & LysoSM(d18:0) & 0.222 & $3.760 \mathrm{E}-07$ \\
\hline HMDB 12420 & $\operatorname{PS}(20: 3 / 16: 0)$ & 0.212 & $2.520 \mathrm{E}-03$ \\
\hline HMDB12634 & 20-COOH-leukotriene E4 & 2.015 & 8.798E-05 \\
\hline HMDB13034 & Palmitoylglycine & 1.802 & $1.660 \mathrm{E}-04$ \\
\hline HMDB13164 & 2-Hydroxylauroylcarnitine & 2.252 & $3.421 \mathrm{E}-06$ \\
\hline HMDB13207 & 9-Hexadecenoylcarnitine & 2.129 & $9.123 \mathrm{E}-04$ \\
\hline HMDB 13250 & Myristoylglycine & 1.902 & $4.683 \mathrm{E}-02$ \\
\hline HMDB 13267 & N-Decanoylglycine & 0.778 & 4.163E-05 \\
\hline HMDB13286 & N-Undecanoylglycine & 0.682 & $2.167 \mathrm{E}-07$ \\
\hline HMDB13303 & Pristanoylglycine & 0.456 & $4.812 \mathrm{E}-07$ \\
\hline HMDB13308 & Stearoylglycine & 1.810 & 4.264E-02 \\
\hline HMDB13317 & Tridecanoylglycine & 4.825 & $9.867 \mathrm{E}-05$ \\
\hline HMDB13327 & Dodecanedioylcarnitine & 2.622 & $6.294 \mathrm{E}-07$ \\
\hline HMDB13331 & 3, 5-Tetradecadiencarnitine & 0.342 & $6.082 \mathrm{E}-05$ \\
\hline HMDB13334 & 9,12-Hexadecadienoylcarnitine & 2.747 & $1.957 \mathrm{E}-03$ \\
\hline HMDB13335 & 3-Hydroxyhexadecadienoylcarnitine & 1.892 & $3.139 \mathrm{E}-08$ \\
\hline HMDB13423 & $\operatorname{PC}(18: 0 / 24: 0)$ & 0.570 & $3.754 \mathrm{E}-05$ \\
\hline HMDB13631 & Oleoyl glycine & 2.163 & 4.413E-06 \\
\hline HMDB28684 & Alanyl-Cysteine & 1.483 & $3.721 \mathrm{E}-04$ \\
\hline HMDB28704 & Arginyl-Asparagine & 1.986 & 7.824E-08 \\
\hline HMDB28728 & Asparaginyl-Cysteine & 1.562 & $2.039 \mathrm{E}-04$ \\
\hline HMDB28789 & Cysteinyl-Gamma-glutamate & 2.422 & $2.714 \mathrm{E}-03$ \\
\hline HMDB28818 & Glutamyl-Glutamate & 1.420 & $1.266 \mathrm{E}-05$ \\
\hline HMDB28829 & Glutamyl-Threonine & 5.395 & $1.550 \mathrm{E}-09$ \\
\hline HMDB28933 & Leucyl-Leucine & 1.485 & $3.272 \mathrm{E}-04$ \\
\hline HMDB28950 & Lysyl-Glutamate & 1.683 & $5.601 \mathrm{E}-03$ \\
\hline HMDB28985 & Methionyl-Tyrosine & 1.822 & $3.664 \mathrm{E}-03$ \\
\hline HMDB29007 & Phenylalanyl-Tyrosine & 1.901 & $2.814 \mathrm{E}-06$ \\
\hline HMDB29087 & Tryptophyl-Leucine & 0.030 & $1.262 \mathrm{E}-04$ \\
\hline HMDB29586 & Octadecylamine & 0.339 & $1.259 \mathrm{E}-0$ \\
\hline
\end{tabular}




\begin{tabular}{llll} 
HMDB38057 & Dehydrophytosphingosine & 1.959 & $6.072 \mathrm{E}-04$ \\
HMDB42660 & TG(14:0/20:4/18:0) & 0.116 & $1.257 \mathrm{E}-07$ \\
HMDB43684 & TG(15:0/18:4/18:0) & 0.081 & $1.914 \mathrm{E}-04$ \\
HMDB59571 & Oxychlordane & 2.501 & $8.717 \mathrm{E}-03$ \\
HMDB59602 & Indanone & 1.825 & $1.646 \mathrm{E}-05$ \\
HMDB59640 & Thio-molybdenum cofactor & 0.487 & $9.587 \mathrm{E}-03$ \\
HMDB59645 & beta-nicotinamide D-ribonucleotide & 2.553 & $1.634 \mathrm{E}-03$ \\
HMDB59744 & 3,4-Methyleneazelaic acid & 0.027 & $1.550 \mathrm{E}-09$ \\
HMDB60185 & Dihomo-gamma-linolenyl coenzyme A & 4.815 & $2.743 \mathrm{E}-02$ \\
HMDB60274 & Sedoheptulose 1,7-bisphosphate & 2.443 & $1.027 \mathrm{E}-06$ \\
HMDB60414 & 6-Methylthiopurine 5'-monophosphate & 1.769 & $5.709 \mathrm{E}-03$ \\
& ribonucleotide & & \\
HMDB61634 & 3-hydroxyoctanoyl carnitine & 0.541 & $7.672 \mathrm{E}-05$ \\
HMDB61639 & 3-hydroxytridecanoyl carnitine & 1.436 & $1.171 \mathrm{E}-05$ \\
HMDB61658 & 3-hydroxyhexadecanoic acid & 1.703 & $1.877 \mathrm{E}-08$ \\
HMDB61690 & 1-Arachidonoylglycerophosphoinositol & 1.762 & $7.265 \mathrm{E}-06$ \\
HMDB61693 & 1-Oleoylglycerophosphoinositol & 1.448 & $6.673 \mathrm{E}-05$ \\
HMDB61695 & 1-Palmitoylglycerophosphoinositol & 1.606 & $1.244 \mathrm{E}-06$ \\
HMDB61696 & 1-Stearoylglycerophosphoinositol/ & 1.480 & $5.987 \mathrm{E}-06$ \\
& 2-Stearoylglycerophosphoinositol & & \\
HMDB61698 & 1-Stearoylglycerophosphoserine & 1.404 & $3.054 \mathrm{E}-04$ \\
\hline
\end{tabular}




\begin{tabular}{|c|c|c|c|}
\hline \multicolumn{4}{|c|}{$\begin{array}{l}\text { Supplementary Table 5. Significantly altered metabolites in BEAS-2B cells } \\
\text { treated with low-dose ZnO NPs. }\end{array}$} \\
\hline ID & Description & Ratio & q value \\
\hline HMDB00034 & Adenine & 0.418 & $6.586 \mathrm{E}-04$ \\
\hline HMDB00050 & Adenosine & 0.443 & $1.980 \mathrm{E}-05$ \\
\hline HMDB00086 & Glycerophosphocholine & 1.306 & $3.370 \mathrm{E}-05$ \\
\hline HMDB00089 & Cytidine & 1.545 & $8.901 \mathrm{E}-03$ \\
\hline HMDB00114 & Glycerylphosphorylethanolamine & 2.116 & $9.630 \mathrm{E}-06$ \\
\hline HMDB00205 & Phenylpyruvic acid & 0.729 & 4.314E-03 \\
\hline HMDB00272 & Phosphoserine & 1.665 & $7.930 \mathrm{E}-04$ \\
\hline HMDB00286 & Uridine diphosphate glucose & 0.309 & $1.690 \mathrm{E}-07$ \\
\hline HMDB00289 & Uric acid & 1.337 & $2.025 \mathrm{E}-02$ \\
\hline HMDB00290 & Uridine diphosphate-N-acetylglucosamine & 1.315 & $3.230 \mathrm{E}-04$ \\
\hline HMDB00296 & Uridine & 0.463 & $1.014 \mathrm{E}-03$ \\
\hline HMDB00300 & Uracil & 0.534 & $2.030 \mathrm{E}-05$ \\
\hline HMDB00517 & L-Arginine & 1.404 & 2.267E-02 \\
\hline HMDB00641 & L-Glutamine & 2.131 & $3.350 \mathrm{E}-05$ \\
\hline HMDB00714 & Hippuric acid & 0.510 & $1.278 \mathrm{E}-03$ \\
\hline HMDB00888 & Undecanedioic acid & 0.454 & $1.236 \mathrm{E}-04$ \\
\hline HMDB00912 & Succinyladenosine & 2.213 & 4.647E-02 \\
\hline HMDB00935 & Uridine diphosphate glucuronic acid & 0.472 & $6.170 \mathrm{E}-05$ \\
\hline HMDB01049 & Gamma-Glutamylcysteine & 0.415 & $3.460 \mathrm{E}-06$ \\
\hline HMDB01066 & S-Lactoylglutathione & 1.526 & $1.245 \mathrm{E}-02$ \\
\hline HMDB01095 & GDP-L-fucose & 1.250 & $1.107 \mathrm{E}-02$ \\
\hline HMDB01138 & N-Acetylglutamic acid & 0.706 & 3.373E-04 \\
\hline HMDB01263 & Allysine & 0.500 & $2.060 \mathrm{E}-02$ \\
\hline HMDB01278 & Presqualene diphosphate & 0.302 & $7.870 \mathrm{E}-05$ \\
\hline HMDB01316 & 6-Phosphogluconic acid & 1.540 & $8.368 \mathrm{E}-04$ \\
\hline HMDB01413 & Citicoline & 0.384 & $1.306 \mathrm{E}-03$ \\
\hline HMDB02030 & Fructosamine & 1.652 & $1.389 \mathrm{E}-03$ \\
\hline HMDB02088 & N-Oleoylethanolamine & 0.404 & $3.306 \mathrm{E}-03$ \\
\hline HMDB02984 & 11-Hydroxyandrosterone & 0.009 & $4.710 \mathrm{E}-11$ \\
\hline HMDB03447 & Tryptophanol & 1.663 & 4.532E-02 \\
\hline HMDB03764 & Glutamylalanine & 1.462 & $6.866 \mathrm{E}-03$ \\
\hline HMDB04220 & Biotinyl-5'-AMP & 1.453 & $1.209 \mathrm{E}-02$ \\
\hline HMDB04976 & Glucosylceramide (d18:1/26:1(17Z)) & 1.849 & $1.927 \mathrm{E}-04$ \\
\hline HMDB06472 & Calcitroic acid & 0.644 & $2.211 \mathrm{E}-02$ \\
\hline HMDB08900 & $\operatorname{PE}(15: 0 / 20: 1)$ & 0.710 & $1.828 \mathrm{E}-02$ \\
\hline HMDB09144 & $\operatorname{PE}(18: 3 / 22: 6)$ & 0.114 & $4.112 \mathrm{E}-03$ \\
\hline HMDB10387 & LysoPC(18:3) & 0.275 & $9.160 \mathrm{E}-08$ \\
\hline HMDB10393 & LysoPC(20:3) & 0.801 & $1.248 \mathrm{E}-02$ \\
\hline HMDB10733 & 3-Oxohexadecanoic acid & 1.213 & $1.449 \mathrm{E}-02$ \\
\hline HMDB11128 & LysoPC(18:0) & 2.099 & $1.316 \mathrm{E}-02$ \\
\hline HMDB11164 & L-beta-aspartyl-L-glutamic acid & 0.399 & $1.131 \mathrm{E}-04$ \\
\hline
\end{tabular}




\begin{tabular}{|c|c|c|c|}
\hline HMDB11171 & L-gamma-glutamyl-L-leucine & 1.612 & $3.870 \mathrm{E}-05$ \\
\hline HMDB11472 & LysoPE(15:0) & 1.605 & $1.740 \mathrm{E}-05$ \\
\hline HMDB11473 & LysoPE(16:0) & 0.830 & 4.223E-02 \\
\hline HMDB11477 & LysoPE(18:2) & 0.781 & $9.853 \mathrm{E}-03$ \\
\hline HMDB11481 & LysoPE(20:0) & 2.026 & $8.663 \mathrm{E}-03$ \\
\hline HMDB11499 & LysoPE(24:6) & 0.482 & $1.690 \mathrm{E}-02$ \\
\hline HMDB11532 & MG(15:0) & 1.351 & 2.711E-02 \\
\hline HMDB11679 & DHAP(6:0) & 1.277 & $1.695 \mathrm{E}-02$ \\
\hline HMDB11720 & UDP-N-acetylmuraminate & 2.070 & $3.763 \mathrm{E}-02$ \\
\hline HMDB11739 & Galactinol dihydrate & 1.826 & $1.152 \mathrm{E}-02$ \\
\hline HMDB12222 & dTDP-4-acetamido-4,6-dideoxy-D-galactose & 1.538 & $1.543 \mathrm{E}-02$ \\
\hline HMDB12420 & $\operatorname{PS}(20: 3 / 16: 0)$ & 0.142 & $9.656 \mathrm{E}-03$ \\
\hline HMDB13034 & Palmitoylglycine & 0.783 & $1.195 \mathrm{E}-03$ \\
\hline HMDB13122 & LysoPC(18:0) & 1.507 & $1.186 \mathrm{E}-02$ \\
\hline HMDB13267 & N-Decanoylglycine & 0.756 & $1.592 \mathrm{E}-04$ \\
\hline HMDB13279 & N-Nonanoylglycine & 0.812 & $3.249 \mathrm{E}-04$ \\
\hline HMDB13286 & N-Undecanoylglycine & 0.717 & $1.890 \mathrm{E}-05$ \\
\hline HMDB13303 & Pristanoylglycine & 0.638 & $7.484 \mathrm{E}-04$ \\
\hline HMDB13317 & Tridecanoylglycine & 2.513 & $3.169 \mathrm{E}-03$ \\
\hline HMDB13423 & $\mathrm{PC}(18: 0 / 24: 0)$ & 0.726 & $3.644 \mathrm{E}-03$ \\
\hline HMDB13631 & Oleoyl glycine & 0.623 & 7.927E-04 \\
\hline HMDB28704 & Arginyl-Asparagine & 1.702 & $3.101 \mathrm{E}-02$ \\
\hline HMDB28720 & Arginyl-Tryptophan & 0.617 & $1.748 \mathrm{E}-04$ \\
\hline HMDB28728 & Asparaginyl-Cysteine & 1.595 & $1.513 \mathrm{E}-04$ \\
\hline HMDB28729 & Asparaginyl-Glutamine & 1.281 & $1.632 \mathrm{E}-02$ \\
\hline HMDB28831 & Glutamyl-Tyrosine & 1.472 & $2.260 \mathrm{E}-05$ \\
\hline HMDB28985 & Methionyl-Tyrosine & 1.475 & $3.885 \mathrm{E}-02$ \\
\hline HMDB29007 & Phenylalanyl-Tyrosine & 1.468 & $3.781 \mathrm{E}-03$ \\
\hline HMDB59601 & Indan-1-ol & 1.745 & $1.302 \mathrm{E}-04$ \\
\hline HMDB59602 & Indanone & 1.785 & $7.050 \mathrm{E}-05$ \\
\hline HMDB59614 & Riboflavin cyclic-4',5'-phosphate & 2.340 & $2.130 \mathrm{E}-06$ \\
\hline HMDB59640 & Thio-molybdenum cofactor & 0.551 & $4.452 \mathrm{E}-02$ \\
\hline HMDB59717 & Glu-Val & 1.756 & $1.278 \mathrm{E}-03$ \\
\hline HMDB59837 & Indane & 1.940 & $7.600 \mathrm{E}-05$ \\
\hline HMDB60244 & Phosphodimethylethanolamine & 1.208 & $1.927 \mathrm{E}-04$ \\
\hline HMDB61634 & 3-hydroxyoctanoyl carnitine & 0.625 & $6.293 \mathrm{E}-04$ \\
\hline
\end{tabular}




\begin{tabular}{|c|c|c|c|}
\hline \multicolumn{4}{|c|}{$\begin{array}{l}\text { Supplementary Table 6. Significantly altered metabolites in BEAS-2B cells } \\
\text { treated with low-dose } \mathrm{SiO}_{2} \text { NPs. }\end{array}$} \\
\hline ID & Description & Ratio & q value \\
\hline HMDB00034 & Adenine & 0.389 & $2.093 \mathrm{E}-05$ \\
\hline HMDB00039 & Butyric acid & 2.662 & $1.275 \mathrm{E}-03$ \\
\hline HMDB00050 & Adenosine & 0.428 & $1.160 \mathrm{E}-05$ \\
\hline HMDB00052 & Argininosuccinic acid & 0.549 & $1.329 \mathrm{E}-07$ \\
\hline HMDB00114 & Glycerylphosphorylethanolamine & 0.435 & $1.788 \mathrm{E}-06$ \\
\hline HMDB00177 & L-Histidine & 0.399 & $3.159 \mathrm{E}-06$ \\
\hline HMDB00195 & Inosine & 0.253 & $3.803 \mathrm{E}-05$ \\
\hline HMDB00201 & L-Acetylcarnitine & 0.379 & $4.769 \mathrm{E}-07$ \\
\hline HMDB00205 & Phenylpyruvic acid & 1.882 & $2.383 \mathrm{E}-05$ \\
\hline HMDB00244 & Riboflavin & 1.394 & $9.417 \mathrm{E}-03$ \\
\hline HMDB00252 & Sphingosine & 6.798 & $2.677 \mathrm{E}-08$ \\
\hline HMDB00277 & Sphingosine 1-phosphate & 1.756 & $8.193 \mathrm{E}-07$ \\
\hline HMDB00280 & Phosphoribosyl pyrophosphate & 1.910 & $1.145 \mathrm{E}-03$ \\
\hline HMDB00286 & Uridine diphosphate glucose & 0.439 & $1.131 \mathrm{E}-06$ \\
\hline HMDB00290 & Uridine diphosphate- $\mathrm{N}$-acetylglucosamine & 0.569 & $2.638 \mathrm{E}-06$ \\
\hline HMDB00623 & Dodecanedioic acid & 1.274 & $6.653 \mathrm{E}-05$ \\
\hline HMDB00656 & Cysteineglutathione disulfide & 2.189 & $2.135 \mathrm{E}-04$ \\
\hline HMDB00672 & Hexadecanedioic acid & 1.430 & $1.532 \mathrm{E}-02$ \\
\hline HMDB00701 & Hexanoylglycine & 1.626 & $8.183 \mathrm{E}-09$ \\
\hline HMDB00779 & Phenyllactic acid & 0.026 & $1.584 \mathrm{E}-10$ \\
\hline HMDB00806 & Myristic acid & 1.567 & $1.353 \mathrm{E}-02$ \\
\hline HMDB00825 & 3'-Sialyllactose/6'-Sialyllactose & 0.100 & $3.829 \mathrm{E}-04$ \\
\hline HMDB00832 & Capryloylglycine & 1.495 & $1.844 \mathrm{E}-05$ \\
\hline HMDB00872 & Tetradecanedioic acid & 1.508 & $2.587 \mathrm{E}-05$ \\
\hline HMDB00888 & Undecanedioic acid & 1.252 & $3.561 \mathrm{E}-04$ \\
\hline HMDB00935 & Uridine diphosphate glucuronic acid & 0.478 & $3.115 \mathrm{E}-05$ \\
\hline HMDB00939 & S-Adenosylhomocysteine & 3.856 & $3.435 \mathrm{E}-04$ \\
\hline HMDB00942 & Tetrahydroneopterin & 0.161 & $4.241 \mathrm{E}-07$ \\
\hline HMDB00943 & Hypoxanthine & 0.144 & $9.708 \mathrm{E}-03$ \\
\hline HMDB00988 & S-Adenosylmethioninamine & 0.466 & $3.369 \mathrm{E}-03$ \\
\hline HMDB00991 & DL-2-Aminooctanoic acid & 0.635 & $1.053 \mathrm{E}-03$ \\
\hline HMDB01043 & Arachidonic acid & 1.631 & $4.608 \mathrm{E}-04$ \\
\hline HMDB01049 & Gamma-Glutamylcysteine & 0.380 & $2.863 \mathrm{E}-07$ \\
\hline HMDB01095 & GDP-L-fucose & 0.688 & $1.470 \mathrm{E}-04$ \\
\hline HMDB01125 & Inositol cyclic phosphate & 0.686 & $2.987 \mathrm{E}-05$ \\
\hline HMDB01142 & FMNH2 & 0.372 & $1.645 \mathrm{E}-05$ \\
\hline HMDB01173 & 5'-Methylthioadenosine & 0.333 & $5.365 \mathrm{E}-10$ \\
\hline HMDB01248 & FAD & 2.376 & $1.595 \mathrm{E}-02$ \\
\hline HMDB01278 & Presqualene diphosphate & 2.179 & $8.602 \mathrm{E}-06$ \\
\hline HMDB01476 & 3-Hydroxyanthranilic acid & 3.158 & $1.753 \mathrm{E}-02$ \\
\hline HMDB01520 & Flavin Mononucleotide & 2.839 & $1.798 \mathrm{E}-03$ \\
\hline
\end{tabular}




\begin{tabular}{|c|c|c|c|}
\hline HMDB01533 & 5,10-Methylene-THF & 0.019 & $2.820 \mathrm{E}-06$ \\
\hline HMDB01553 & 2-Oxo-4-methylthiobutanoic acid & 0.743 & $1.057 \mathrm{E}-05$ \\
\hline HMDB01570 & Thymidine 3',5'-cyclic monophosphate & 2.089 & $3.322 \mathrm{E}-05$ \\
\hline HMDB01846 & Tetrahydrofolic acid & 0.064 & $1.831 \mathrm{E}-03$ \\
\hline HMDB01976 & Docosapentaenoic acid (22n-6) & 2.169 & $2.839 \mathrm{E}-05$ \\
\hline HMDB02012 & Ubiquinone-1 & 0.099 & $6.952 \mathrm{E}-12$ \\
\hline HMDB02014 & cis-5-Tetradecenoylcarnitine & 0.545 & $7.684 \mathrm{E}-07$ \\
\hline HMDB02030 & Fructosamine & 1.783 & $5.540 \mathrm{E}-04$ \\
\hline HMDB02088 & N-Oleoylethanolamine & 2.870 & $1.241 \mathrm{E}-04$ \\
\hline HMDB02095 & Malonylcarnitine & 0.042 & $3.566 \mathrm{E}-05$ \\
\hline HMDB02206 & Molybdopterin & 5.396 & $7.335 \mathrm{E}-04$ \\
\hline HMDB02334 & Benzoquinoneacetic acid & 3.660 & $3.762 \mathrm{E}-03$ \\
\hline HMDB02639 & Sulfolithocholylglycine & 0.537 & $3.633 \mathrm{E}-03$ \\
\hline HMDB02664 & Prostaglandin E2 & 2.210 & $3.859 \mathrm{E}-04$ \\
\hline HMDB02757 & Cysteic acid & 1.549 & $1.290 \mathrm{E}-02$ \\
\hline HMDB02823 & Docosatrienoic acid & 2.426 & $2.452 \mathrm{E}-03$ \\
\hline HMDB02984 & 11-Hydroxyandrosterone & 0.111 & $2.862 \mathrm{E}-07$ \\
\hline HMDB03229 & Palmitoleic acid & 0.701 & $1.503 \mathrm{E}-03$ \\
\hline HMDB03231 & Vaccenic acid & 1.638 & $5.686 \mathrm{E}-05$ \\
\hline HMDB03536 & dIDP & 0.411 & $1.145 \mathrm{E}-04$ \\
\hline HMDB03601 & (S)-Reticuline & 2.813 & $2.897 \mathrm{E}-07$ \\
\hline HMDB03752 & LysoPC(10:0) & 9.751 & $5.442 \mathrm{E}-05$ \\
\hline HMDB04231 & Pantothenol & 0.081 & $5.844 \mathrm{E}-08$ \\
\hline HMDB04866 & Lactosylceramide (d18:1/12:0) & 0.195 & $1.819 \mathrm{E}-06$ \\
\hline HMDB04976 & Glucosylceramide (d18:1/26:1(17Z)) & 0.012 & $3.177 \mathrm{E}-04$ \\
\hline HMDB05060 & Eicosadienoic acid & 1.972 & $2.134 \mathrm{E}-04$ \\
\hline HMDB06555 & dIMP & 0.605 & 7.630E-04 \\
\hline HMDB06752 & Dihydroceramide & 11.520 & $3.348 \mathrm{E}-06$ \\
\hline HMDB06944 & 1,4-beta-D-Glucan & 0.415 & 8.649E-04 \\
\hline HMDB07877 & $\operatorname{PC}(14: 0 / 18: 4)$ & 0.403 & $1.437 \mathrm{E}-04$ \\
\hline HMDB07940 & $\operatorname{PC}(15: 0 / 18: 2)$ & 22.969 & $1.805 \mathrm{E}-03$ \\
\hline HMDB07961 & $\operatorname{PC}(15: 0 / 16: 0)$ & 5.023 & $1.043 \mathrm{E}-02$ \\
\hline HMDB08457 & PC(20:4/P-18:1) & 2.679 & $8.856 \mathrm{E}-06$ \\
\hline HMDB08786 & $\mathrm{PC}(24: 0 / \mathrm{P}-18: 1)$ & 0.387 & $5.256 \mathrm{E}-04$ \\
\hline HMDB08834 & $\mathrm{PE}(14: 0 / 20: 1)$ & 2.686 & $2.485 \mathrm{E}-07$ \\
\hline HMDB09542 & $\operatorname{PE}(22: 1 / 24: 1)$ & 0.103 & $5.821 \mathrm{E}-04$ \\
\hline HMDB09603 & $\operatorname{PE}(22: 4 / 22: 5)$ & 5.178 & $9.082 \mathrm{E}-12$ \\
\hline HMDB10331 & Palmitoyl glucuronide & 1.898 & $2.741 \mathrm{E}-04$ \\
\hline HMDB10337 & 6-Dehydrotestosterone glucuronide & 3.943 & 2.709E-04 \\
\hline HMDB10364 & 11-Hydroxyprogesterone 11-glucuronide & 5.442 & $9.269 \mathrm{E}-05$ \\
\hline HMDB 10378 & 5,8,11-Eicosatrienoic acid & 1.748 & $1.053 \mathrm{E}-05$ \\
\hline HMDB10379 & LysoPC(14:0) & 0.625 & $1.620 \mathrm{E}-03$ \\
\hline HMDB 10380 & LysoPC(14:1) & 0.672 & $2.835 \mathrm{E}-06$ \\
\hline HMDB10399 & LysoPC(22:1) & 3.378 & $2.453 \mathrm{E}-08$ \\
\hline
\end{tabular}




\begin{tabular}{|c|c|c|c|}
\hline HMDB10405 & LysoPC(24:0) & 1.971 & $1.110 \mathrm{E}-03$ \\
\hline HMDB10674 & PG(18:3/22:6) & 4.520 & $6.415 \mathrm{E}-06$ \\
\hline HMDB10733 & 3-Oxohexadecanoic acid & 1.871 & $1.553 \mathrm{E}-05$ \\
\hline HMDB11153 & MG(P-18:0e/0:0/0:0) & 1.947 & $5.325 \mathrm{E}-$ \\
\hline HMDB11177 & L-phenylalanyl-L-proline & 0.147 & $6.464 \mathrm{E}-0$ \\
\hline HMDB 11470 & LysoPE(14:0) & 0.700 & $5.030 \mathrm{E}-05$ \\
\hline HMDB 11473 & LysoPE(0:0/16:0) & 3.077 & $1.276 \mathrm{E}-09$ \\
\hline HMDB11474 & LysoPE(16:1) & 0.663 & $1.568 \mathrm{E}-07$ \\
\hline HMDB11475 & LysoPE(18:1) & 0.744 & $6.011 \mathrm{E}-06$ \\
\hline HMDB 11477 & LysoPE(18:2) & 0.713 & $8.535 \mathrm{E}-\mathrm{C}$ \\
\hline HMDB11532 & $\operatorname{MG}(15: 0)$ & 1.552 & $1.141 \mathrm{E}-02$ \\
\hline HMDB11541 & $\operatorname{MG}(18: 4)$ & 1.720 & $6.141 \mathrm{E}-04$ \\
\hline HMDB11610 & 5-Taurinomethyl-2-thiouridine & 1.637 & $6.323 \mathrm{E}-0$ \\
\hline HMDB11627 & Farnesylcysteine & 0.681 & $1.478 \mathrm{E}-\mathrm{C}$ \\
\hline HMDB11684 & $\mathrm{N}(6)-($ Octanoyl)lysine & 0.025 & $1.090 \mathrm{E}-03$ \\
\hline HMDB11759 & Cer(d18:0/14:0) & 10.107 & $2.558 \mathrm{E}-06$ \\
\hline HMDB11760 & $\operatorname{Cer}(\mathrm{d} 18: 0 / 16: 0)$ & 9.459 & $3.092 \mathrm{E}-0$ \\
\hline HMDB12082 & LysoSM(d18:0) & 0.157 & $2.153 \mathrm{E}-1$ \\
\hline HMDB12305 & UDP-L-rhamnose & 1.501 & $6.000 \mathrm{E}-\mathrm{C}$ \\
\hline HMDB12634 & 20-COOH-leukotriene E4 & 0.604 & $1.203 \mathrm{E}-\mathrm{C}$ \\
\hline HMDB12932 & Dynorphin A (6-8) & 0.567 & $3.328 \mathrm{E}-0$ \\
\hline HMDB 13010 & N-Heptanoylglycine & 1.264 & $6.149 \mathrm{E}-0$ \\
\hline HMDB13207 & 9-Hexadecenoylcarnitine & 0.304 & $3.323 \mathrm{E}-\mathrm{C}$ \\
\hline HMDB 13250 & Myristoylglycine & 3.400 & $4.047 \mathrm{E}-0$ \\
\hline HMDB13286 & N-Undecanoylglycine & 0.741 & $6.189 \mathrm{E}-0$ \\
\hline HMDB13287 & $\mathrm{Ne}, \mathrm{Ne}$ dimethyllysine & 0.674 & $2.194 \mathrm{E}-0$ \\
\hline HMDB13303 & Pristanoylglycine & 0.588 & $3.127 \mathrm{E}-\mathrm{C}$ \\
\hline HMDB 13308 & Stearoylglycine & 0.785 & $1.254 \mathrm{E}-03$ \\
\hline HMDB13317 & Tridecanoylglycine & 8.090 & $2.105 \mathrm{E}-0$ \\
\hline HMDB13327 & Dodecanedioylcarnitine & 2.087 & $8.662 \mathrm{E}-0$ \\
\hline HMDB 13330 & 3-Hydroxy-cis-5-tetradecenoylcarnitine & 0.628 & $4.504 \mathrm{E}-0$ \\
\hline HMDB13331 & 3, 5-Tetradecadiencarnitine & 0.426 & $5.125 \mathrm{E}-0$ \\
\hline HMDB13334 & 9,12-Hexadecadienoylcarnitine & 0.482 & $1.439 \mathrm{E}-0$ \\
\hline HMDB13335 & 3-Hydroxyhexadecadienoylcarnitine & 0.572 & $1.469 \mathrm{E}-\mathrm{C}$ \\
\hline HMDB13631 & Oleoyl glycine & 0.386 & 8.339E-0 \\
\hline HMDB28720 & Arginyl-Tryptophan & 0.449 & $2.239 \mathrm{E}-0$ \\
\hline HMDB28761 & Aspartyl-Proline & 0.688 & $2.000 \mathrm{E}-0$ \\
\hline HMDB28763 & Aspartyl-Threonine & 0.563 & $1.881 \mathrm{E}-0$ \\
\hline HMDB28789 & Cysteinyl-Gamma-glutamate & 4.128 & $2.928 \mathrm{E}-0$ \\
\hline HMDB28829 & Glutamyl-Threonine & 6.233 & $3.205 \mathrm{E}-\mathrm{C}$ \\
\hline HMDB28950 & Lysyl-Glutamate & 2.045 & $7.901 \mathrm{E}-05$ \\
\hline HMDB28985 & Methionyl-Tyrosine & 1.843 & $1.934 \mathrm{E}-0$ \\
\hline HMDB29007 & Phenylalanyl-Tyrosine & 1.915 & $1.481 \mathrm{E}-\mathrm{C}$ \\
\hline HMDB29087 & Tryptophyl-Leucine & 0.049 & $9.549 \mathrm{E}-0$ \\
\hline
\end{tabular}




\begin{tabular}{llll} 
HMDB29097 & Tryptophyl-Gamma-glutamate & 0.373 & $5.559 \mathrm{E}-04$ \\
HMDB29586 & Octadecylamine & 0.324 & $3.409 \mathrm{E}-06$ \\
HMDB38057 & Dehydrophytosphingosine & 3.423 & $2.329 \mathrm{E}-05$ \\
HMDB41623 & N6-Carbamoyl-L-threonyladenosine & 0.629 & $8.644 \mathrm{E}-03$ \\
HMDB42660 & TG(14:0/20:4/18:0) & 0.191 & $3.527 \mathrm{E}-12$ \\
HMDB42720 & TG(14:0/22:4/18:0) & 4.079 & $2.232 \mathrm{E}-03$ \\
HMDB43684 & TG(15:0/18:4/18:0) & 0.182 & $1.955 \mathrm{E}-05$ \\
HMDB59571 & Oxychlordane & 4.706 & $1.007 \mathrm{E}-10$ \\
HMDB59614 & Riboflavin cyclic-4',5'-phosphate & 2.797 & $1.817 \mathrm{E}-06$ \\
HMDB59744 & 3,4-Methyleneazelaic acid & 0.021 & $1.457 \mathrm{E}-12$ \\
HMDB60129 & 24-Oxo-1alpha,23,25-trihydroxyvitamin & 3.006 & $2.971 \mathrm{E}-11$ \\
& D3 & & \\
HMDB60179 & 3-Sulfinato-L-alaninate & 1.755 & $5.870 \mathrm{E}-05$ \\
HMDB60830 & Melatonin glucuronide & 2.042 & $6.685 \mathrm{E}-03$ \\
HMDB61522 & PE(MonoMe(11,3)/MonoMe(11,5)) & 4.063 & $4.273 \mathrm{E}-03$ \\
HMDB61636 & 3-hydroxydecanoyl carnitine & 2.266 & $2.168 \mathrm{E}-03$ \\
HMDB61639 & 3-hydroxytridecanoyl carnitine & 1.692 & $2.064 \mathrm{E}-07$ \\
HMDB61641 & 3-hydroxypentadecanoyl carnitine & 2.223 & $2.279 \mathrm{E}-05$ \\
HMDB61653 & 3-hydroxyheptanoic acid & 1.759 & $1.930 \mathrm{E}-03$ \\
HMDB61658 & 3-hydroxyhexadecanoic acid & 1.580 & $5.525 \mathrm{E}-07$ \\
HMDB61661 & 9-hydroxyoctadecanoic acid & 1.309 & $9.877 \mathrm{E}-03$ \\
HMDB61692 & 1-Linoleoylglycerophosphocholine/ & 4.832 & $7.690 \mathrm{E}-10$ \\
& 2-Linoleoylglycerophosphocholine & & \\
\hline & & & \\
\hline
\end{tabular}




\begin{tabular}{|c|c|c|c|}
\hline \multicolumn{4}{|c|}{$\begin{array}{l}\text { Supplementary Table 7. Significantly altered metabolites in BEAS-2B cells } \\
\text { treated with low-dose } \mathrm{TiO}_{2} \text { NPs. }\end{array}$} \\
\hline ID & Description & Ratio & q value \\
\hline HMDB00039 & Butyric acid & 20.239 & $5.173 \mathrm{E}-06$ \\
\hline HMDB00052 & Argininosuccinic acid & 28.233 & $3.195 \mathrm{E}-08$ \\
\hline HMDB00114 & Glycerylphosphorylethanolamine & 0.292 & $6.312 \mathrm{E}-08$ \\
\hline HMDB00201 & L-Acetylcarnitine & 0.272 & 3.894E-06 \\
\hline HMDB00205 & Phenylpyruvic acid & 2.357 & $1.774 \mathrm{E}-06$ \\
\hline HMDB00244 & Riboflavin & 1.624 & $1.401 \mathrm{E}-04$ \\
\hline HMDB00252 & Sphingosine & 6.710 & $6.172 \mathrm{E}-08$ \\
\hline HMDB00277 & Sphingosine 1-phosphate & 1.320 & $3.377 \mathrm{E}-03$ \\
\hline HMDB00286 & Uridine diphosphate glucose & 0.132 & $2.290 \mathrm{E}-09$ \\
\hline HMDB00290 & Uridine diphosphate- $\mathrm{N}$-acetylglucosamine & 0.548 & $6.590 \mathrm{E}-07$ \\
\hline HMDB00296 & Uridine & 3.226 & $1.994 \mathrm{E}-05$ \\
\hline HMDB00300 & Uracil & 3.049 & $5.496 \mathrm{E}-09$ \\
\hline HMDB00403 & 2-Hydroxyadenine & 1.980 & $1.411 \mathrm{E}-06$ \\
\hline HMDB00779 & Phenyllactic acid & 0.027 & $7.316 \mathrm{E}-15$ \\
\hline HMDB00806 & Myristic acid & 1.514 & $3.055 \mathrm{E}-02$ \\
\hline HMDB00825 & 3'-Sialyllactose/6'-Sialyllactose & 0.212 & $3.289 \mathrm{E}-06$ \\
\hline HMDB00872 & Tetradecanedioic acid & 1.390 & $1.114 \mathrm{E}-04$ \\
\hline HMDB00935 & Uridine diphosphate glucuronic acid & 0.145 & $3.313 \mathrm{E}-08$ \\
\hline HMDB00939 & S-Adenosylhomocysteine & 3.178 & $8.576 \mathrm{E}-04$ \\
\hline HMDB00942 & Tetrahydroneopterin & 0.185 & $9.856 \mathrm{E}-06$ \\
\hline HMDB01043 & Arachidonic acid & 2.252 & $9.170 \mathrm{E}-06$ \\
\hline HMDB01095 & GDP-L-fucose & 0.640 & $1.091 \mathrm{E}-04$ \\
\hline HMDB01125 & Inositol cyclic phosphate & 0.750 & $1.091 \mathrm{E}-04$ \\
\hline HMDB01373 & Dephospho-CoA & 2.114 & $1.373 \mathrm{E}-02$ \\
\hline HMDB01416 & Pantetheine 4'-phosphate & 0.538 & $1.793 \mathrm{E}-05$ \\
\hline HMDB01520 & Flavin Mononucleotide & 3.760 & $3.043 \mathrm{E}-04$ \\
\hline HMDB01533 & 5,10-Methylene-THF & 0.017 & 8.779E-09 \\
\hline HMDB01846 & Tetrahydrofolic acid & 0.075 & $7.055 \mathrm{E}-04$ \\
\hline HMDB01976 & Docosapentaenoic acid (22n-6) & 2.590 & $2.902 \mathrm{E}-06$ \\
\hline HMDB02012 & Ubiquinone-1 & 0.096 & $3.654 \mathrm{E}-11$ \\
\hline HMDB02030 & Fructosamine & 2.044 & $1.424 \mathrm{E}-03$ \\
\hline HMDB02183 & Docosahexaenoic acid & 1.778 & $2.061 \mathrm{E}-03$ \\
\hline HMDB02224 & 5-Methyldeoxycytidine & 30.422 & $4.300 \mathrm{E}-06$ \\
\hline HMDB02334 & Benzoquinoneacetic acid & 10.847 & $5.720 \mathrm{E}-04$ \\
\hline HMDB02343 & 5,6-DHET & 3.100 & $7.607 \mathrm{E}-14$ \\
\hline HMDB02664 & Prostaglandin E2 & 1.928 & $2.320 \mathrm{E}-03$ \\
\hline HMDB02823 & Docosatrienoic acid & 2.333 & $5.244 \mathrm{E}-03$ \\
\hline HMDB02984 & 11-Hydroxyandrosterone & 0.440 & $9.100 \mathrm{E}-06$ \\
\hline HMDB03229 & Palmitoleic acid & 2.874 & $1.327 \mathrm{E}-04$ \\
\hline HMDB03231 & Vaccenic acid & 1.772 & $3.124 \mathrm{E}-05$ \\
\hline HMDB03577 & VPGPR Enterostatin & 3.621 & $8.405 \mathrm{E}-06$ \\
\hline
\end{tabular}




\begin{tabular}{|c|c|c|c|}
\hline HMDB03601 & (S)-Reticuline & 30.493 & 8.779E-09 \\
\hline HMDB03752 & LysoPC(10:0) & 7.757 & 4.119E-04 \\
\hline HMDB04047 & Adipoyl-CoA & 0.605 & $2.925 \mathrm{E}-02$ \\
\hline HMDB04231 & Pantothenol & 0.082 & $1.916 \mathrm{E}-07$ \\
\hline HMDB04708 & 9,12,13-TriHOME & 1.502 & $1.059 \mathrm{E}-02$ \\
\hline HMDB04866 & Lactosylceramide (d18:1/12:0) & 0.127 & $7.439 \mathrm{E}-06$ \\
\hline HMDB04985 & Aspartylysine & 2.343 & $5.001 \mathrm{E}-07$ \\
\hline HMDB05060 & Eicosadienoic acid & 2.256 & $4.429 \mathrm{E}-06$ \\
\hline HMDB05862 & 2-Methylguanosine & 0.531 & $5.457 \mathrm{E}-05$ \\
\hline HMDB06236 & Phenylacetaldehyde & 17.382 & $2.812 \mathrm{E}-13$ \\
\hline HMDB06353 & Dolichol phosphate & 4.531 & $3.252 \mathrm{E}-04$ \\
\hline HMDB06472 & Calcitroic acid & 1.950 & $1.473 \mathrm{E}-03$ \\
\hline HMDB06752 & Dihydroceramide & 8.234 & $1.449 \mathrm{E}-06$ \\
\hline HMDB07877 & $\operatorname{PC}(14: 0 / 18: 4)$ & 0.169 & $9.719 \mathrm{E}-07$ \\
\hline HMDB08156 & $\mathrm{PC}(18: 2 / 22: 6)$ & 0.214 & $5.388 \mathrm{E}-05$ \\
\hline HMDB08457 & PC(20:4/P-18:1) & 2.005 & $4.980 \mathrm{E}-05$ \\
\hline HMDB08834 & $\mathrm{PE}(14: 0 / 20: 1)$ & 3.986 & $1.612 \mathrm{E}-07$ \\
\hline HMDB08845 & $\operatorname{PE}(14: 0 / 22: 5)$ & 2.950 & $3.365 \mathrm{E}-08$ \\
\hline HMDB09603 & $\operatorname{PE}(22: 4 / 22: 5)$ & 20.624 & $2.598 \mathrm{E}-15$ \\
\hline HMDB09819 & $\operatorname{PI}(18: 0 / 22: 5)$ & 15.461 & $9.883 \mathrm{E}-03$ \\
\hline HMDB10337 & 6-Dehydrotestosterone glucuronide & 5.897 & $1.349 \mathrm{E}-04$ \\
\hline HMDB10364 & 11-Hydroxyprogesterone 11-glucuronide & 8.187 & $6.248 \mathrm{E}-06$ \\
\hline HMDB10378 & 5,8,11-Eicosatrienoic acid & 2.229 & $1.603 \mathrm{E}-06$ \\
\hline HMDB10380 & LysoPC(14:1) & 3.839 & 4.708E-06 \\
\hline HMDB10397 & LysoPC(20:5) & 2.590 & $6.071 \mathrm{E}-08$ \\
\hline HMDB10399 & LysoPC(22:1) & 18.043 & $1.780 \mathrm{E}-12$ \\
\hline HMDB10404 & LysoPC(22:6) & 2.284 & $3.751 \mathrm{E}-06$ \\
\hline HMDB10733 & 3-Oxohexadecanoic acid & 1.737 & $3.717 \mathrm{E}-06$ \\
\hline HMDB11177 & L-phenylalanyl-L-proline & 0.136 & $3.007 \mathrm{E}-07$ \\
\hline HMDB11470 & LysoPE(14:0) & 0.638 & $1.024 \mathrm{E}-04$ \\
\hline HMDB11471 & LysoPE(14:1) & 1.380 & $1.370 \mathrm{E}-03$ \\
\hline HMDB11473 & LysoPE(16:0) & 15.304 & $1.088 \mathrm{E}-15$ \\
\hline HMDB11474 & LysoPE(16:1) & 0.634 & 4.684E-08 \\
\hline HMDB11475 & LysoPE(18:1) & 0.748 & $1.279 \mathrm{E}-03$ \\
\hline HMDB11477 & LysoPE(18:2) & 0.722 & $1.270 \mathrm{E}-05$ \\
\hline HMDB11478 & LysoPE(18:3) & 0.725 & $2.453 \mathrm{E}-06$ \\
\hline HMDB11484 & LysoPE(20:3) & 0.683 & $1.093 \mathrm{E}-03$ \\
\hline HMDB11487 & LysoPE(20:4) & 0.691 & $2.425 \mathrm{E}-05$ \\
\hline HMDB11489 & LysoPE(20:5) & 0.709 & $5.212 \mathrm{E}-05$ \\
\hline HMDB11493 & LysoPE(22:4) & 0.753 & $8.172 \mathrm{E}-03$ \\
\hline HMDB11494 & LysoPE(22:5) & 0.760 & $2.649 \mathrm{E}-03$ \\
\hline HMDB11499 & LysoPE(24:6) ) & 0.551 & $3.442 \mathrm{E}-02$ \\
\hline HMDB11513 & LysoPE(20:2) & 0.677 & $2.460 \mathrm{E}-03$ \\
\hline HMDB11526 & LysoPE(22:6) & 0.735 & $9.187 \mathrm{E}-06$ \\
\hline
\end{tabular}




\begin{tabular}{|c|c|c|c|}
\hline HMDB11532 & MG(15:0) & 1.850 & $6.530 \mathrm{E}-03$ \\
\hline HMDB11541 & MG(18:4) & 1.715 & $6.685 \mathrm{E}-06$ \\
\hline HMDB11627 & Farnesylcysteine & 0.624 & $1.413 \mathrm{E}-07$ \\
\hline HMDB11684 & $\mathrm{N}(6)-($ Octanoyl)lysine & 0.033 & $3.032 \mathrm{E}-03$ \\
\hline HMDB11739 & Galactinol dihydrate & 1.905 & $4.008 \mathrm{E}-03$ \\
\hline HMDB12082 & LysoSM(d18:0) & 0.176 & 4.475E-09 \\
\hline HMDB12420 & $\operatorname{PS}(20: 3 / 16: 0)$ & 0.217 & $2.913 \mathrm{E}-06$ \\
\hline HMDB13250 & Myristoylglycine & 0.284 & $2.912 \mathrm{E}-05$ \\
\hline HMDB13286 & N-Undecanoylglycine & 0.696 & $2.138 \mathrm{E}-07$ \\
\hline HMDB13303 & Pristanoylglycine & 0.424 & $1.774 \mathrm{E}-06$ \\
\hline HMDB13317 & Tridecanoylglycine & 5.778 & $5.782 \mathrm{E}-05$ \\
\hline HMDB13327 & Dodecanedioylcarnitine & 2.790 & $3.673 \mathrm{E}-07$ \\
\hline HMDB13331 & 3, 5-Tetradecadiencarnitine & 0.394 & 4.187E-04 \\
\hline HMDB28755 & Aspartyl-Histidine & 22.719 & $1.099 \mathrm{E}-03$ \\
\hline HMDB28829 & Glutamyl-Threonine & 6.663 & $9.736 \mathrm{E}-12$ \\
\hline HMDB28893 & Histidinyl-Proline & 4.690 & $9.137 \mathrm{E}-04$ \\
\hline HMDB28985 & Methionyl-Tyrosine & 1.849 & $2.523 \mathrm{E}-03$ \\
\hline HMDB29007 & Phenylalanyl-Tyrosine & 2.226 & $8.300 \mathrm{E}-07$ \\
\hline HMDB29087 & Tryptophyl-Leucine & 0.041 & $1.910 \mathrm{E}-02$ \\
\hline HMDB29586 & Octadecylamine & 0.307 & $6.058 \mathrm{E}-06$ \\
\hline HMDB38057 & Dehydrophytosphingosine & 2.181 & $1.607 \mathrm{E}-04$ \\
\hline HMDB42036 & Thymidine glycol & 1.536 & $2.939 \mathrm{E}-05$ \\
\hline HMDB42660 & TG(14:0/20:4/18:0) & 0.107 & $2.058 \mathrm{E}-06$ \\
\hline HMDB43684 & TG(15:0/18:4/18:0) & 0.158 & $2.133 \mathrm{E}-04$ \\
\hline HMDB59571 & Oxychlordane & 37.415 & 4.153E-12 \\
\hline HMDB59614 & Riboflavin cyclic-4',5'-phosphate & 1.777 & $6.815 \mathrm{E}-05$ \\
\hline HMDB59645 & beta-nicotinamide D-ribonucleotide & 2.325 & $2.380 \mathrm{E}-03$ \\
\hline HMDB59729 & 3,4-Methylenesebacic acid & 3.968 & $1.243 \mathrm{E}-08$ \\
\hline HMDB59744 & 3,4-Methyleneazelaic acid & 0.023 & $2.789 \mathrm{E}-11$ \\
\hline HMDB59759 & 1-Hydroxypyrene glucuronide & 4.039 & 7.514E-04 \\
\hline HMDB60129 & $\begin{array}{l}\text { 24-Oxo-1alpha,23,25-trihydroxyvitamin } \\
\text { D3 }\end{array}$ & 12.831 & $2.282 \mathrm{E}-13$ \\
\hline HMDB60274 & Sedoheptulose 1,7-bisphosphate & 1.824 & $2.297 \mathrm{E}-03$ \\
\hline HMDB61522 & PE(MonoMe(11,3)/MonoMe(11,5)) & 6.131 & $1.500 \mathrm{E}-03$ \\
\hline HMDB61641 & 3-hydroxypentadecanoyl carnitine & 3.165 & $1.177 \mathrm{E}-07$ \\
\hline HMDB61658 & 3-hydroxyhexadecanoic acid & 1.572 & $1.687 \mathrm{E}-08$ \\
\hline HMDB61692 & $\begin{array}{l}\text { 1-Linoleoylglycerophosphocholine/ } \\
\text { 2-Linoleoylglycerophosphocholine }\end{array}$ & 35.674 & $1.641 \mathrm{E}-11$ \\
\hline
\end{tabular}




\begin{tabular}{|c|c|c|c|}
\hline \multicolumn{4}{|c|}{$\begin{array}{l}\text { Supplementary Table 8. Significantly altered metabolites in BEAS-2B cells } \\
\text { treated with low-dose } \mathrm{CeO}_{2} \text { NPs. }\end{array}$} \\
\hline ID & Description & Ratio & q value \\
\hline HMDB00089 & Cytidine & 1.592 & $9.460 \mathrm{E}-03$ \\
\hline HMDB00099 & L-Cystathionine & 1.853 & 4.291E-03 \\
\hline HMDB00114 & Glycerylphosphorylethanolamine & 0.311 & $7.292 \mathrm{E}-04$ \\
\hline HMDB00201 & L-Acetylcarnitine & 0.156 & $2.402 \mathrm{E}-08$ \\
\hline HMDB00205 & Phenylpyruvic acid & 2.205 & $4.336 \mathrm{E}-06$ \\
\hline HMDB00244 & Riboflavin & 1.522 & $1.299 \mathrm{E}-03$ \\
\hline HMDB00252 & Sphingosine & 7.044 & $4.978 \mathrm{E}-06$ \\
\hline HMDB00272 & Phosphoserine & 0.628 & $1.839 \mathrm{E}-04$ \\
\hline HMDB00277 & Sphingosine 1-phosphate & 1.434 & $5.028 \mathrm{E}-04$ \\
\hline HMDB00286 & Uridine diphosphate glucose & 0.126 & $2.796 \mathrm{E}-09$ \\
\hline HMDB00290 & Uridine diphosphate- $\mathrm{N}$-acetylglucosamine & 0.481 & $1.053 \mathrm{E}-07$ \\
\hline HMDB00296 & Uridine & 5.591 & $1.041 \mathrm{E}-06$ \\
\hline HMDB00300 & Uracil & 5.495 & $2.068 \mathrm{E}-08$ \\
\hline HMDB00403 & 2-Hydroxyadenine & 2.017 & $8.754 \mathrm{E}-06$ \\
\hline HMDB00630 & Cytosine & 1.691 & $7.412 \mathrm{E}-07$ \\
\hline HMDB00701 & Hexanoylglycine & 1.387 & 9.944E-04 \\
\hline HMDB00705 & Hexanoylcarnitine & 0.691 & $2.772 \mathrm{E}-02$ \\
\hline HMDB00779 & Phenyllactic acid & 0.029 & $2.577 \mathrm{E}-13$ \\
\hline HMDB00806 & Myristic acid & 1.666 & 8.007E-03 \\
\hline HMDB00825 & 3'-Sialyllactose/6'-Sialyllactose & 0.309 & $1.193 \mathrm{E}-05$ \\
\hline HMDB00888 & Undecanedioic acid & 0.374 & $6.695 \mathrm{E}-06$ \\
\hline HMDB00912 & Succinyladenosine & 10.876 & $1.070 \mathrm{E}-04$ \\
\hline HMDB00935 & Uridine diphosphate glucuronic acid & 0.115 & 4.848E-08 \\
\hline HMDB00939 & S-Adenosylhomocysteine & 3.224 & $6.608 \mathrm{E}-04$ \\
\hline HMDB00942 & Tetrahydroneopterin & 0.223 & 4.144E-04 \\
\hline HMDB00962 & Lipoamide & 1.897 & $1.219 \mathrm{E}-02$ \\
\hline HMDB01043 & Arachidonic acid & 2.529 & $1.409 \mathrm{E}-05$ \\
\hline HMDB01049 & Gamma-Glutamylcysteine & 2.460 & $1.849 \mathrm{E}-06$ \\
\hline HMDB01095 & GDP-L-fucose & 0.675 & $2.547 \mathrm{E}-02$ \\
\hline HMDB01125 & Inositol cyclic phosphate & 0.656 & $9.880 \mathrm{E}-05$ \\
\hline HMDB01179 & Nicotinic acid adenine dinucleotide & 0.593 & $2.226 \mathrm{E}-02$ \\
\hline HMDB01272 & Nicotine glucuronide & 1.485 & $3.808 \mathrm{E}-06$ \\
\hline HMDB01373 & Dephospho-CoA & 1.899 & $1.726 \mathrm{E}-02$ \\
\hline HMDB01413 & Citicoline & 4.191 & 3.902E-05 \\
\hline HMDB01520 & Flavin Mononucleotide & 5.480 & $2.654 \mathrm{E}-04$ \\
\hline HMDB01533 & 5,10-Methylene-THF & 0.026 & $6.885 \mathrm{E}-08$ \\
\hline HMDB01570 & Thymidine 3',5'-cyclic monophosphate & 1.980 & $2.912 \mathrm{E}-04$ \\
\hline HMDB01846 & Tetrahydrofolic acid & 0.127 & $2.362 \mathrm{E}-04$ \\
\hline HMDB01976 & Docosapentaenoic acid (22n-6) & 2.806 & $9.482 \mathrm{E}-07$ \\
\hline HMDB02007 & Tetracosahexaenoic acid & 0.702 & $1.681 \mathrm{E}-04$ \\
\hline HMDB02012 & Ubiquinone-1 & 0.103 & $2.116 \mathrm{E}-11$ \\
\hline
\end{tabular}




\begin{tabular}{|c|c|c|c|}
\hline HMDB02030 & Fructosamine & 1.839 & $2.533 \mathrm{E}-03$ \\
\hline HMDB02135 & S-(3-oxo-3-carboxy-n-propyl)cysteine & 1.828 & 7.939E-03 \\
\hline HMDB02183 & Docosahexaenoic acid & 1.820 & $2.370 \mathrm{E}-03$ \\
\hline HMDB02334 & Benzoquinoneacetic acid & 3.206 & $5.604 \mathrm{E}-03$ \\
\hline HMDB02664 & Prostaglandin E2 & 2.464 & $7.572 \mathrm{E}-05$ \\
\hline HMDB02817 & N-Acetylglucosamine 6-phosphate & 1.858 & $7.255 \mathrm{E}-07$ \\
\hline HMDB02823 & Docosatrienoic acid & 2.101 & $2.647 \mathrm{E}-02$ \\
\hline HMDB02984 & 11-Hydroxyandrosterone & 0.025 & $1.425 \mathrm{E}-10$ \\
\hline HMDB03134 & Biocytin & 0.630 & $9.771 \mathrm{E}-06$ \\
\hline HMDB03229 & Palmitoleic acid & 2.969 & $1.831 \mathrm{E}-04$ \\
\hline HMDB03231 & Vaccenic acid & 1.912 & $3.363 \mathrm{E}-05$ \\
\hline HMDB03351 & GDP-glucose & 0.526 & $1.518 \mathrm{E}-05$ \\
\hline HMDB03447 & Tryptophanol & 1.913 & $9.876 \mathrm{E}-04$ \\
\hline HMDB03752 & LysoPC(10:0) & 6.728 & 5.795E-04 \\
\hline HMDB03976 & D-Glucuronic acid 1-phosphate & 0.728 & $1.987 \mathrm{E}-05$ \\
\hline HMDB04047 & Adipoyl-CoA & 0.420 & $1.780 \mathrm{E}-02$ \\
\hline HMDB04231 & Pantothenol & 0.086 & $1.869 \mathrm{E}-06$ \\
\hline HMDB04985 & Aspartylysine & 3.062 & $3.276 \mathrm{E}-07$ \\
\hline HMDB05060 & Eicosadienoic acid & 2.069 & $2.272 \mathrm{E}-03$ \\
\hline HMDB06049 & O-Phosphotyrosine & 0.505 & $6.580 \mathrm{E}-04$ \\
\hline HMDB06472 & Calcitroic acid & 1.797 & $3.038 \mathrm{E}-03$ \\
\hline HMDB06752 & Dihydroceramide & 8.081 & $5.603 \mathrm{E}-06$ \\
\hline HMDB07877 & $\mathrm{PC}(14: 0 / 18: 4)$ & 0.065 & 5.713E-05 \\
\hline HMDB08156 & $\mathrm{PC}(18: 2 / 22: 6)$ & 0.068 & $3.764 \mathrm{E}-04$ \\
\hline HMDB09144 & $\operatorname{PE}(18: 3 / 22: 6)$ & 0.171 & $4.929 \mathrm{E}-05$ \\
\hline HMDB09739 & $\operatorname{PE}(24: 0 / 24: 0)$ & 0.310 & $1.037 \mathrm{E}-02$ \\
\hline HMDB09773 & $\operatorname{PE}(24: 1 / 24: 1)$ & 0.627 & $6.959 \mathrm{E}-06$ \\
\hline HMDB10331 & Palmitoyl glucuronide & 1.645 & $1.973 \mathrm{E}-03$ \\
\hline HMDB10337 & 6-Dehydrotestosterone glucuronide & 2.337 & $1.817 \mathrm{E}-03$ \\
\hline HMDB10364 & 11-Hydroxyprogesterone 11-glucuronide & 3.471 & $3.070 \mathrm{E}-04$ \\
\hline HMDB10378 & 5,8,11-Eicosatrienoic acid & 2.213 & $4.138 \mathrm{E}-07$ \\
\hline HMDB10387 & LysoPC(18:3) & 2.832 & $1.049 \mathrm{E}-08$ \\
\hline HMDB10674 & PG(18:3/22:6) & 2.697 & $1.295 \mathrm{E}-04$ \\
\hline HMDB10733 & 3-Oxohexadecanoic acid & 1.634 & $1.729 \mathrm{E}-0$ \\
\hline HMDB11153 & MG(P-18:0e/0:0/0:0) & 1.498 & $2.488 \mathrm{E}-06$ \\
\hline HMDB11164 & L-beta-aspartyl-L-glutamic acid & 3.577 & $3.851 \mathrm{E}-09$ \\
\hline HMDB11177 & L-phenylalanyl-L-proline & 0.155 & $1.488 \mathrm{E}-0$ \\
\hline HMDB11470 & LysoPE(14:0) & 0.663 & $9.971 \mathrm{E}-06$ \\
\hline HMDB11471 & LysoPE(14:1) & 1.348 & $1.416 \mathrm{E}-03$ \\
\hline HMDB11473 & LysoPE(16:0) & 0.687 & $1.813 \mathrm{E}-0$ \\
\hline HMDB11474 & LysoPE(16:1) & 0.649 & $1.306 \mathrm{E}-07$ \\
\hline HMDB11477 & LysoPE(18:2) & 0.750 & $5.381 \mathrm{E}-05$ \\
\hline HMDB11478 & LysoPE(18:3) & 0.728 & $2.143 \mathrm{E}-\mathrm{C}$ \\
\hline HMDB11480 & LysoPE(18:4) & 1.383 & $6.572 \mathrm{E}-\mathrm{C}$ \\
\hline
\end{tabular}




\begin{tabular}{|c|c|c|c|}
\hline HMDB11484 & LysoPE(20:3) & 0.706 & $2.329 \mathrm{E}-03$ \\
\hline HMDB11487 & LysoPE(20:4) & 0.750 & $6.802 \mathrm{E}-05$ \\
\hline HMDB11489 & LysoPE(20:5) & 0.726 & 2.149E-04 \\
\hline HMDB11513 & LysoPE(20:2) & 0.703 & $6.322 \mathrm{E}-03$ \\
\hline HMDB11532 & $\operatorname{MG}(15: 0)$ & 1.437 & $2.705 \mathrm{E}-02$ \\
\hline HMDB11538 & $\operatorname{MG}(0: 0 / 18: 2(9 Z, 12 Z) / 0: 0)$ & 0.672 & $2.219 \mathrm{E}-04$ \\
\hline HMDB11541 & $\operatorname{MG}(18: 4)$ & 1.997 & $2.786 \mathrm{E}-05$ \\
\hline HMDB11545 & $\operatorname{MG}(20: 3)$ & 0.723 & 8.007E-05 \\
\hline HMDB11627 & Farnesylcysteine & 0.666 & $1.161 \mathrm{E}-06$ \\
\hline HMDB11684 & $\mathrm{N}(6)-($ Octanoyl)lysine & 0.036 & $7.916 \mathrm{E}-06$ \\
\hline HMDB11687 & Phenylbutyrylglutamine & 2.563 & $2.165 \mathrm{E}-06$ \\
\hline HMDB11739 & Galactinol dihydrate & 3.185 & $3.090 \mathrm{E}-04$ \\
\hline HMDB12082 & LysoSM(d18:0) & 0.169 & $6.988 \mathrm{E}-10$ \\
\hline HMDB12188 & Presqualene diphosphate & 0.376 & $1.163 \mathrm{E}-02$ \\
\hline HMDB12420 & $\operatorname{PS}(20: 3 / 16: 0)$ & 0.309 & $1.556 \mathrm{E}-03$ \\
\hline HMDB 13250 & Myristoylglycine & 1.289 & $3.139 \mathrm{E}-05$ \\
\hline HMDB13267 & N-Decanoylglycine & 0.797 & $1.909 \mathrm{E}-07$ \\
\hline HMDB13286 & N-Undecanoylglycine & 0.695 & $3.986 \mathrm{E}-07$ \\
\hline HMDB13303 & Pristanoylglycine & 0.498 & $1.859 \mathrm{E}-06$ \\
\hline HMDB13317 & Tridecanoylglycine & 4.516 & $1.650 \mathrm{E}-04$ \\
\hline HMDB13330 & 3-Hydroxy-cis-5-tetradecenoylcarnitine & 0.714 & $5.202 \mathrm{E}-05$ \\
\hline HMDB13331 & 3, 5-Tetradecadiencarnitine & 0.458 & $2.038 \mathrm{E}-03$ \\
\hline HMDB13423 & $\mathrm{PC}(18: 0 / 24: 0)$ & 0.495 & 7.703E-06 \\
\hline HMDB28696 & Alanyl-Serine & 1.819 & $3.541 \mathrm{E}-03$ \\
\hline HMDB28704 & Arginyl-Asparagine & 1.482 & 4.057E-02 \\
\hline HMDB28728 & Asparaginyl-Cysteine & 1.619 & $1.099 \mathrm{E}-04$ \\
\hline HMDB28789 & Cysteinyl-Gamma-glutamate & 8.807 & $8.482 \mathrm{E}-0$ \\
\hline HMDB28829 & Glutamyl-Threonine & 5.667 & $1.521 \mathrm{E}-09$ \\
\hline HMDB28934 & Leucyl-Lysine & 0.408 & $1.614 \mathrm{E}-04$ \\
\hline HMDB28950 & Lysyl-Glutamate & 1.932 & $2.133 \mathrm{E}-04$ \\
\hline HMDB28985 & Methionyl-Tyrosine & 1.802 & $3.405 \mathrm{E}-03$ \\
\hline HMDB29007 & Phenylalanyl-Tyrosine & 1.659 & $2.502 \mathrm{E}-05$ \\
\hline HMDB29087 & Tryptophyl-Leucine & 0.022 & $5.050 \mathrm{E}-03$ \\
\hline HMDB38057 & Dehydrophytosphingosine & 2.486 & $2.986 \mathrm{E}-04$ \\
\hline HMDB42036 & Thymidine glycol & 2.140 & $2.832 \mathrm{E}-07$ \\
\hline HMDB42660 & TG(14:0/20:4/18:0) & 0.068 & $2.806 \mathrm{E}-05$ \\
\hline HMDB43684 & TG(15:0/18:4/18:0) & 0.117 & $1.680 \mathrm{E}-05$ \\
\hline HMDB59571 & Oxychlordane & 2.808 & $5.061 \mathrm{E}-03$ \\
\hline HMDB59602 & Indanone & 1.858 & $1.556 \mathrm{E}-05$ \\
\hline HMDB59640 & Thio-molybdenum cofactor & 0.519 & $1.446 \mathrm{E}-0$ \\
\hline HMDB59645 & beta-nicotinamide D-ribonucleotide & 2.350 & $1.027 \mathrm{E}-0$ \\
\hline HMDB59744 & 3,4-Methyleneazelaic acid & 0.025 & $1.171 \mathrm{E}-09$ \\
\hline HMDB60185 & Dihomo-gamma-linolenyl coenzyme A & 4.565 & $3.990 \mathrm{E}-0$ \\
\hline HMDB60244 & Phosphodimethylethanolamine & 0.734 & $1.977 \mathrm{E}-\mathrm{C}$ \\
\hline
\end{tabular}


HMDB60274 Sedoheptulose 1,7-bisphosphate ribonucleotide

HMDB60830 Melatonin glucuronide

HMDB61658 3-hydroxyhexadecanoic acid
$1.566 \quad 3.173 \mathrm{E}-03$

$1.591 \quad 2.832 \mathrm{E}-07$

